\title{
Recent Research on Jensen's Inequality for Oparators
}

\author{
Jadranka Mićić1 and Josip Pečarić2 \\ Additional information is available at the end of the chapter \\ http://dx.doi.org/10.5772/48468
}

\section{Introduction}

The self-adjoint operators on Hilbert spaces with their numerous applications play an important part in the operator theory. The bounds research for self-adjoint operators is a very useful area of this theory. There is no better inequality in bounds examination than Jensen's inequality. It is an extensively used inequality in various fields of mathematics.

Let $I$ be a real interval of any type. A continuous function $f: I \rightarrow \mathbb{R}$ is said to be operator convex if

$$
f(\lambda x+(1-\lambda) y) \leq \lambda f(x)+(1-\lambda) f(y)
$$

holds for each $\lambda \in[0,1]$ and every pair of self-adjoint operators $x$ and $y$ (acting) on an infinite dimensional Hilbert space $H$ with spectra in $I$ (the ordering is defined by setting $x \leq y$ if $y-x$ is positive semi-definite).

Let $f$ be an operator convex function defined on an interval $I$. Ch. Davis [1] provedThere is small typo in the proof. Davis states that $\phi$ by Stinespring's theorem can be written on the form $\phi(x)=P \rho(x) P$ where $\rho$ is a ${ }^{*}$-homomorphism to $B(H)$ and $P$ is a projection on $H$. In fact, $H$ may be embedded in a Hilbert space $K$ on which $\rho$ and $P$ acts. The theorem then follows by the calculation $f(\phi(x))=f(P \rho(x) P) \leq P f(\rho(x)) P=P \rho(f(x) P=\phi(f(x))$, where the pinching inequality, proved by Davis in the same paper, is applied. a Schwarz inequality

$$
f(\phi(x)) \leq \phi(f(x))
$$

where $\phi: \rightarrow B(K)$ is a unital completely positive linear mapping from a $C^{*}$-algebra to linear operators on a Hilbert space $K$, and $x$ is a self-adjoint element in with spectrum in $I$. Subsequently M. D. Choi [2] noted that it is enough to assume that $\phi$ is unital and positive. In 
fact, the restriction of $\phi$ to the commutative $C^{*}$-algebra generated by $x$ is automatically completely positive by a theorem of Stinespring.

F. Hansen and G. K. Pedersen [3] proved a Jensen type inequality

$$
f\left(\sum_{i=1}^{n} a_{i}^{*} x_{i} a_{i}\right) \leq \sum_{i=1}^{n} a_{i}^{*} f\left(x_{i}\right) a_{i}
$$

for operator convex functions $f$ defined on an interval $I=[0, \alpha$ ) (with $\alpha \leq \infty$ and $f(0) \leq 0$ ) and self-adjoint operators $x_{1}, \cdots, x_{n}$ with spectra in $I$ assuming that $\sum_{i=1}^{n} a_{i}^{*} a_{i}=\mathbf{1}$. The restriction on the interval and the requirement $f(0) \leq 0$ was subsequently removed by B. Mond and J. Pečarić in [4], cf. also [5].

The inequality (ㅁ) is in fact just a reformulation of (ㅁ) although this was not noticed at the time. It is nevertheless important to note that the proof given in [3] and thus the statement of the theorem, when restricted to $n \times n$ matrices, holds for the much richer class of $2 n \times 2 n$ matrix convex functions. Hansen and Pedersen used ( $\square$ ) to obtain elementary operations on functions, which leave invariant the class of operator monotone functions. These results then served as the basis for a new proof of Löwner's theorem applying convexity theory and Krein-Milman's theorem.

B. Mond and J. Pečarić [6] proved the inequality

$$
f\left(\sum_{i=1}^{n} w_{i} \phi_{i}\left(x_{i}\right)\right) \leq \sum_{i=1}^{n} w_{i} \phi_{i}\left(f\left(x_{i}\right)\right)
$$

for operator convex functions $f$ defined on an interval $I$, where $\phi_{i}: B(H) \rightarrow B(K)$ are unital positive linear mappings, $x_{1}, \cdots, x_{n}$ are self-adjoint operators with spectra in $I$ and $w_{1}, \cdots, w_{n}$ are are non-negative real numbers with sum one.

Also, B. Mond, J. Pečarić, T. Furuta et al. [6], [7], [8], [9], [10], [11] observed conversed of some special case of Jensen's inequality. So in [10] presented the following generalized converse of a Schwarz inequality (口)

$$
F[\phi(f(A)), g(\phi(A))] \leq \max _{m \leq t \leq M} F\left[f(m)+\frac{f(M)-f(m)}{M-m}(t-m), g(t)\right] 1_{\tilde{n}}
$$

for convex functions $f$ defined on an interval $[m, M], m<M$, where $g$ is a real valued continuous function on $[m, M], F(u, v)$ is a real valued function defined on $U \times V$, matrix nondecreasing in $u, U \supset f[m, M], V \supset g[m, M], \phi: H_{n} \rightarrow H_{\tilde{n}}$ is a unital positive linear mapping and $A$ is a Hermitian matrix with spectrum contained in $[m, M]$.

There are a lot of new research on the classical Jensen inequality ( $\square$ ) and its reverse inequalities. For example, J.I. Fujii et all. in [12], [13] expressed these inequalities by externally dividing points. 


\section{Classic results}

In this section we present a form of Jensen's inequality which contains (ㅁ), (ㅁ) and (ㅁ) as special cases. Since the inequality in $(\square)$ was the motivating step for obtaining converses of Jensen's inequality using the so-called Mond-Pečarić method, we also give some results pertaining to converse inequalities in the new formulation.

We recall some definitions. Let $T$ be a locally compact Hausdorff space and let be a $C^{*}$-algebra of operators on some Hilbert space $H$. We say that a field $\left(x_{t}\right)_{t \in T}$ of operators in is continuous if the function $t \mapsto x_{t}$ is norm continuous on $T$. If in addition $\mu$ is a Radon measure on $T$ and the function $t \mapsto\left\|x_{t}\right\|$ is integrable, then we can form the Bochner integral $\int_{T} x_{t} d \mu(t)$, which is the unique element in such that

$$
\varphi\left(\int_{T} x_{t} d \mu(t)\right)=\int_{T} \varphi\left(x_{t}\right) d \mu(t)
$$

for every linear functional $\varphi$ in the norm dual ${ }^{*}$.

Assume furthermore that there is a field $\left(\phi_{t}\right)_{t \in T}$ of positive linear mappings $\phi_{t}: \rightarrow \mathcal{B}$ from to another "-algebra $\mathcal{B}$ of operators on a Hilbert space $K$. We recall that a linear mapping $\phi_{t}: \rightarrow \mathcal{B}$ is said to be a positive mapping if $\phi_{t}\left(x_{t}\right) \geq 0$ for all $x_{t} \geq 0$. We say that such a field is continuous if the function $t \mapsto \phi_{t}(x)$ is continuous for every $x \in$. Let the *algebras include the identity operators and the function $t \mapsto \phi_{t}\left(1_{H}\right)$ be integrable with $\int_{T} \phi_{t}\left(1_{H}\right) d \mu(t)=k 1_{K}$ for some positive scalar $k$. Specially, if $\int_{T} \phi_{t}\left(1_{H}\right) d \mu(t)=1_{K}$, we say that a field $\left(\phi_{t}\right)_{t \in T}$ is unital.

Let $B(H)$ be the $C^{*}$-algebra of all bounded linear operators on a Hilbert space $H$. We define bounds of an operator $x \in B(H)$ by

$$
m_{x}=\inf _{\|\xi\|=1}\langle x \xi, \xi\rangle \text { and } M_{x}=\sup _{\|\xi\|=1}\langle x \xi, \xi\rangle
$$

for $\xi \in H$. If $(x)$ denotes the spectrum of $x$, then $(x) \subseteq\left[m_{x}, M_{x}\right]$.

For an operator $x \in B(H)$ we define operators $|x|, x^{+}, x^{-}$by

$$
|x|=\left(x^{*} x\right)^{1 / 2}, \quad x^{+}=(|x|+x) / 2, \quad x^{-}=(|x|-x) / 2
$$

Obviously, if $x$ is self-adjoint, then $|x|=\left(x^{2}\right)^{1 / 2}$ and $x^{+}, x^{-} \geq 0$ (called positive and negative parts of $\left.x=x^{+}-x^{-}\right)$. 


\subsection{Jensen's inequality with operator convexity}

Firstly, we give a general formulation of Jensen's operator inequality for a unital field of positive linear mappings (see [14]).

Theorem 1 Let $f: I \rightarrow \mathbb{R}$ be an operator convex function defined on an interval $I$ and let and $\mathcal{B}$ be unital $C^{*}$-algebras acting on a Hilbert space $H$ and $K$ respectively. If $\left(\phi_{t}\right)_{t \in T}$ is a unital field of positive linear mappings $\phi_{t}: \rightarrow \mathcal{B}$ defined on a locally compact Hausdorff space $T$ with a bounded Radon measure $\mu$, then the inequality

$$
f\left(\int_{T} \phi_{t}\left(x_{t}\right) d \mu(t)\right) \leq \int_{T} \phi_{t}\left(f\left(x_{t}\right)\right) d \mu(t)
$$

holds for every bounded continuous field $\left(x_{t}\right)_{t \in T}$ of self-adjoint elements in with spectra contained in $I$.

We first note that the function $t \mapsto \phi_{t}\left(x_{t}\right) \in \mathcal{B}$ is continuous and bounded, hence integrable with respect to the bounded Radon measure $\mu$. Furthermore, the integral is an element in the multiplier algebra $M(\mathcal{B})$ acting on $K$. We may organize the set $C B(T$,$) of bounded con-$ tinuous functions on $T$ with values in as a normed involutive algebra by applying the point-wise operations and setting

$$
\left\|\left(y_{t}\right)_{t \in T}\right\|=\sup _{t \in T} \quad\left\|y_{t}\right\| \quad\left(y_{t}\right)_{t \in T} \in C B(T,)
$$

and it is not difficult to verify that the norm is already complete and satisfy the $C^{*}$-identity. In fact, this is a standard construction in $C^{*}$-algebra theory. It follows that $f\left(\left(x_{t}\right)_{t \in T}\right)=\left(f\left(x_{t}\right)\right)_{t \in T}$. We then consider the mapping

$$
\pi: C B(T,) \rightarrow M(\mathcal{B}) \subseteq B(K)
$$

defined by setting

$$
\pi\left(\left(x_{t}\right)_{t \in T}\right)=\int_{T} \phi_{t}\left(x_{t}\right) d \mu(t)
$$

and note that it is a unital positive linear map. Setting $x=\left(x_{t}\right)_{t \in T} \in C B(T$,$) , we use in-$ equality $(\square)$ to obtain

$$
f\left(\pi\left(\left(x_{t}\right)_{t \in T}\right)\right)=f(\pi(x)) \leq \pi(f(x))=\pi\left(f\left(\left(x_{t}\right)_{t \in T}\right)\right)=\pi\left(\left(f\left(x_{t}\right)\right)_{t \in T}\right)
$$

but this is just the statement of the theorem. 


\subsection{Converses of Jensen's inequality}

In the present context we may obtain results of the Li-Mathias type cf. Chapter 3[15] and [16], [17].

Theorem 2 Let $T$ be a locally compact Hausdorff space equipped with a bounded Radon measure $\mu$. Let $\left(x_{t}\right)_{t \in T}$ be a bounded continuous field of self-adjoint elements in a unital $C^{*}$ algebra with spectra in $[m, M], m<M$. Furthermore, let $\left(\phi_{t}\right)_{t \in T}$ be a field of positive linear mappings $\phi_{t}: \rightarrow \mathcal{B}$ from to another unital $C^{*}$ - algebra $\mathcal{B}$, such that the function $t \mapsto \phi_{t}\left(1_{H}\right)$ is integrable with $\int_{T} \phi_{t}\left(1_{H}\right) d \mu(t)=k 1_{K}$ for some positive scalar $k$. Let $m_{x}$ and $M_{x}, m_{x} \leq M_{x}$, be the bounds of the self-adjoint operator $x=\int_{T} \phi_{t}\left(x_{t}\right) d \mu(t)$ and $f:[m, M] \rightarrow \mathbb{R}$, $g:\left[m_{x}, M_{x}\right] \rightarrow \mathbb{R}, F: U \times V \rightarrow \mathbb{R}$ be functions such that $(k f)([m, M]) \subset U, g\left(\left[m_{x}, M_{x}\right]\right) \subset V$ and $F$ is bounded. If $F$ is operator monotone in the first variable, then

$$
\begin{gathered}
\inf _{m_{x} \leq z \leq M_{x}} F\left[k \cdot h_{1}\left(\frac{1}{k} z\right), g(z)\right] 1_{K} \leq F\left[\int_{T} \phi_{t}\left(f\left(x_{t}\right)\right) d \mu(t), g\left(\int_{T} \phi_{t}\left(x_{t}\right) d \mu(t)\right)\right] \\
\leq \sup _{m_{x} \leq z \leq M_{x}} F\left[k \cdot h_{2}\left(\frac{1}{k} z\right), g(z)\right] 1_{K}
\end{gathered}
$$

holds for every operator convex function $h_{1}$ on $[m, M]$ such that $h_{1} \leq f$ and for every operator concave function $h_{2}$ on $[m, M]$ such that $h_{2} \geq f$.

We prove only RHS of $(\square)$. Let $h_{2}$ be operator concave function on $[m, M]$ such that $f(z) \leq h_{2}(z)$ for every $z \in[m, M]$. By using the functional calculus, it follows that $f\left(x_{t}\right) \leq h_{2}\left(x_{t}\right)$ for every $t \in T$. Applying the positive linear mappings $\phi_{t}$ and integrating, we obtain

$$
\int_{T} \phi_{t}\left(f\left(x_{t}\right)\right) d \mu(t) \leq \int_{T} \phi_{t}\left(h_{2}\left(x_{t}\right)\right) d \mu(t)
$$

Furthermore, replacing $\phi_{t}$ by $\frac{1}{k} \phi_{t}$ in Theorem $\square$, we obtain $\frac{1}{k} \int_{T} \phi_{t}\left(h_{2}\left(x_{t}\right)\right) d \mu(t) \leq h_{2}\left(\frac{1}{k} \int_{T} \phi_{t}\left(x_{t}\right) d \mu(t)\right), \quad$ which gives $\int_{T} \phi_{t}\left(f\left(x_{t}\right)\right) d \mu(t) \leq k \cdot h_{2}\left(\frac{1}{k} \int_{T} \phi_{t}\left(x_{t}\right) d \mu(t)\right)$. Since $m_{x} 1_{K} \leq \int_{T} \phi_{t}\left(x_{t}\right) d \mu(t) \leq M_{x} 1_{K}$, then using operator monotonicity of $F(\cdot, v)$ we obtain

$$
\begin{gathered}
F\left[\int_{T} \phi_{t}\left(f\left(x_{t}\right)\right) d \mu(t), g\left(\int_{T} \phi_{t}\left(x_{t}\right) d \mu(t)\right)\right] \\
\leq F\left[k \cdot h_{2}\left(\frac{1}{k} \int_{T} \phi_{t}\left(x_{t}\right) d \mu(t)\right), g\left(\int_{T} \phi_{t}\left(x_{t}\right) d \mu(t)\right)\right] \leq \sup _{m_{x} \leq z \leq M_{x}} F\left[k \cdot h_{2}\left(\frac{1}{k} z\right), g(z)\right] 1_{K}
\end{gathered}
$$


Applying RHS of (口) for a convex function $f$ (or LHS of ( $\square$ ) for a concave function $f$ ) we obtain the following generalization of $(\square)$.

Theorem 3 Let $\left(x_{t}\right)_{t \in T}, m_{x}, M_{x}$ and $\left(\phi_{t}\right)_{t \in T}$ be as in Theorem $\square$. Let $f:[m, M] \rightarrow \mathbb{R}$, $g:\left[m_{x}, M_{x}\right] \rightarrow \mathbb{R}, F: U \times V \rightarrow \mathbb{R}$ be functions such that $(k f)([m, M]) \subset U, g\left(\left[m_{x}, M_{x}\right]\right) \subset V$ and $F$ is bounded. If $F$ is operator monotone in the first variable and $f$ is convex on the interval $[m, M]$, then

$$
\begin{gathered}
F\left[\int_{T} \phi_{t}\left(f\left(x_{t}\right)\right) d \mu(t), g\left(\int_{T} \phi_{t}\left(x_{t}\right) d \mu(t)\right)\right] \\
\leq \sup _{m_{x} \leq z \leq M_{x}} F\left[\frac{M k-z}{M-m} f(m)+\frac{z-k m}{M-m} f(M), g(z)\right] 1_{K}
\end{gathered}
$$

In the dual case (when $f$ is concave) the opposite inequalities hold in ( $\square$ ) with inf instead of sup.

We prove only the convex case. For convex $f$ the inequality $f(z) \leq \frac{M-z}{M-m} f(m)+\frac{z-m}{M-m} f(M)$ holds for every $z \in[m, M]$. Thus, by putting $h_{2}(z)=\frac{M-z}{M-m} f(m)+\frac{z-m}{M-m} f(M)$ in (口) we obtain (口). Numerous applications of the previous theorem can be given (see [15]). Applying Theorem $\square$ for the function $F(u, v)=u-\alpha v$ and $k=1$, we obtain the following generalization of Theorem 2.4[15].

Corollary 4 Let $\left(x_{t}\right)_{t \in T}, m_{x}, M_{x}$ be as in Theorem $\square$ and $\left(\phi_{t}\right)_{t \in T}$ be a unital field of positive linear mappings $\phi_{t}: \rightarrow \mathcal{B}$. If $f:[m, M] \rightarrow \mathbb{R}$ is convex on the interval $[m, M], m<M$, and $g:[m, M] \rightarrow \mathbb{R}$, then for any $\alpha \in \mathbb{R}$

$$
\int_{T} \phi_{t}\left(f\left(x_{t}\right)\right) d \mu(t) \leq \alpha g\left(\int_{T} \phi_{t}\left(x_{t}\right) d \mu(t)\right)+C 1_{K}
$$

where

$$
\begin{aligned}
C & =\max _{m_{x} \leq z \leq M_{x}}\left\{\frac{M-z}{M-m} f(m)+\frac{z-m}{M-m} f(M)-\alpha g(z)\right\} \\
& \leq \max _{m \leq z \leq M}\left\{\frac{M-z}{M-m} f(m)+\frac{z-m}{M-m} f(M)-\alpha g(z)\right\}
\end{aligned}
$$

If furthermore $\alpha g$ is strictly convex differentiable, then the constant $C \equiv C(m, M, f, g, \alpha)$ can be written more precisely as

$$
C=\frac{M-z_{0}}{M-m} f(m)+\frac{z_{0}-m}{M-m} f(M)-\alpha g\left(z_{0}\right)
$$

where 


$$
z_{0}= \begin{cases}g^{\prime-1}\left(\frac{f(M)-f(m)}{\alpha(M-m)}\right) & \text { if } \quad \alpha g^{\prime}\left(m_{x}\right) \leq \frac{f(M)-f(m)}{M-m} \leq \alpha g^{\prime}\left(M_{x}\right) \\ m_{x} & \text { if } \alpha g^{\prime}\left(m_{x}\right) \geq \frac{f(M)-f(m)}{M-m} \\ M_{x} & \text { if } \alpha g^{\prime}\left(M_{x}\right) \leq \frac{f(M)-f(m)}{M-m}\end{cases}
$$

In the dual case (when $f$ is concave and $\alpha g$ is strictly concave differentiable) the opposite inequalities hold in (ㅁ) with min instead of max with the opposite condition while deter$\operatorname{mining} z_{0}$.

\section{Inequalities with conditions on spectra}

In this section we present Jensens's operator inequality for real valued continuous convex functions with conditions on the spectra of the operators. A discrete version of this result is given in [18]. Also, we obtain generalized converses of Jensen's inequality under the same conditions.

Operator convexity plays an essential role in (ㄷ). In fact, the inequality ( $\square$ ) will be false if we replace an operator convex function by a general convex function. For example, M.D. Choi in Remark 2.6[2] considered the function $f(t)=t^{4}$ which is convex but not operator convex. He demonstrated that it is sufficient to put $\operatorname{dim} H=3$, so we have the matrix case as follows. Let $\Phi: M_{3}(\mathbb{C}) \rightarrow M_{2}(\mathbb{C})$ be the contraction mapping $\Phi\left(\left(a_{i j}\right)_{1 \leq i, j \leq 3}\right)=\left(a_{i j}\right)_{1 \leq i, j \leq 2}$. If $A=\left(\begin{array}{lll}1 & 0 & 1 \\ 0 & 0 & 1 \\ 1 & 1 & 1\end{array}\right)$, then $\Phi(A)^{4}=\left(\begin{array}{ll}1 & 0 \\ 0 & 0\end{array}\right) \neg \leq\left(\begin{array}{ll}9 & 5 \\ 5 & 3\end{array}\right)=\Phi\left(A^{4}\right)$ and no relation between $\Phi(A)^{4}$ and $\Phi\left(A^{4}\right)$ under the operator order.

Example 5 It appears that the inequality (ㄷ) will be false if we replace the operator convex function by a general convex function. We give a small example for the matrix cases and $T=\{1,2\}$. We define mappings $\Phi_{1}, \Phi_{2}: M_{3}(\mathbb{C}) \rightarrow M_{2}(\mathbb{C})$ by $\Phi_{1}\left(\left(a_{i j}\right)_{1 \leq i, j \leq 3}\right)=\frac{1}{2}\left(a_{i j}\right)_{1 \leq i, j \leq 2^{\prime}}$ $\Phi_{2}=\Phi_{1}$. Then $\Phi_{1}\left(I_{3}\right)+\Phi_{2}\left(I_{3}\right)=I_{2}$.

I)

- If

$$
X_{1}=2\left(\begin{array}{lll}
1 & 0 & 1 \\
0 & 0 & 1 \\
1 & 1 & 1
\end{array}\right) \text { and } X_{2}=2\left(\begin{array}{lll}
1 & 0 & 0 \\
0 & 0 & 0 \\
0 & 0 & 0
\end{array}\right)
$$

then 


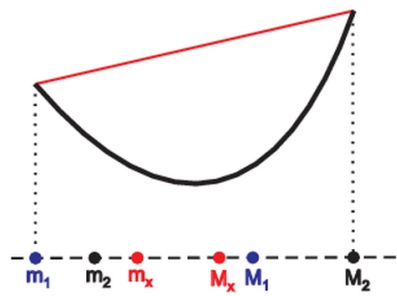

a)

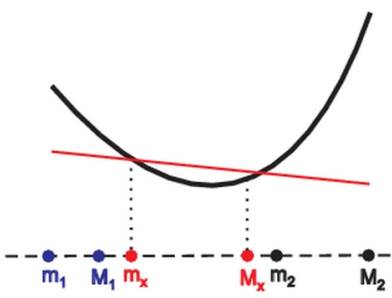

b)

Figure 1. Spectral conditions for a convex function $f$

$$
\left(\Phi_{1}\left(X_{1}\right)+\Phi_{2}\left(X_{2}\right)\right)^{4}=\left(\begin{array}{cc}
16 & 0 \\
0 & 0
\end{array}\right) \neg \leq\left(\begin{array}{cc}
80 & 40 \\
40 & 24
\end{array}\right)=\Phi_{1}\left(X_{1}^{4}\right)+\Phi_{2}\left(X_{2}^{4}\right)
$$

Given the above, there is no relation between $\left(\Phi_{1}\left(X_{1}\right)+\Phi_{2}\left(X_{2}\right)\right)^{4}$ and $\Phi_{1}\left(X_{1}^{4}\right)+\Phi_{2}\left(X_{2}^{4}\right)$ under the operator order. We observe that in the above case the following stands $X=\Phi_{1}\left(X_{1}\right)+\Phi_{2}\left(X_{2}\right)=\left(\begin{array}{ll}2 & 0 \\ 0 & 0\end{array}\right) \quad$ and $\quad\left[m_{x}, M_{x}\right]=[0,2], \quad\left[m_{1}, M_{1}\right] \subset[-1.60388,4.49396]$, $\left[m_{2}, M_{2}\right]=[0,2]$, i.e.

$$
\left(m_{x}, M_{x}\right) \subset\left[m_{1}, M_{1}\right] \cup\left[m_{2}, M_{2}\right]
$$

(see Fig. 1.a).

II)

- If

$$
X_{1}=\left(\begin{array}{ccc}
-14 & 0 & 1 \\
0 & -2 & -1 \\
1 & -1 & -1
\end{array}\right) \text { and } X_{2}=\left(\begin{array}{ccc}
15 & 0 & 0 \\
0 & 2 & 0 \\
0 & 0 & 15
\end{array}\right)
$$

then

$$
\left(\Phi_{1}\left(X_{1}\right)+\Phi_{2}\left(X_{2}\right)\right)^{4}=\left(\begin{array}{cc}
\frac{1}{16} & 0 \\
0 & 0
\end{array}\right)<\left(\begin{array}{cc}
89660 & -247 \\
-247 & 51
\end{array}\right)=\Phi_{1}\left(X_{1}^{4}\right)+\Phi_{2}\left(X_{2}^{4}\right)
$$

So we have that an inequality of type (־) now is valid. In the above case the following stands $\quad X=\Phi_{1}\left(X_{1}\right)+\Phi_{2}\left(X_{2}\right)=\left(\begin{array}{cc}\frac{1}{2} & 0 \\ 0 & 0\end{array}\right) \quad$ and $\quad\left[m_{x}, M_{x}\right]=[0,0.5]$, $\left[m_{1}, M_{1}\right] \subset[-14.077,-0.328566],\left[m_{2}, M_{2}\right]=[2,15]$, i.e. 


$$
\left(m_{x}, M_{x}\right) \cap\left[m_{1}, M_{1}\right]=\varnothing \quad \text { and } \quad\left(m_{x}, M_{x}\right) \cap\left[m_{2}, M_{2}\right]=\varnothing
$$

(see Fig. 1.b).

\subsection{Jensen's inequality without operator convexity}

It is no coincidence that the inequality (ㅁ) is valid in Example $\square-\mathrm{II})$. In the following theorem we prove a general result when Jensen's operator inequality ( $\square$ ) holds for convex functions.

Theorem 6 Let $\left(x_{t}\right)_{t \in T}$ be a bounded continuous field of self-adjoint elements in a unital $C^{*}$ algebra defined on a locally compact Hausdorff space $T$ equipped with a bounded Radon measure $\mu$. Let $m_{t}$ and $M_{t}, m_{t} \leq M_{t}$, be the bounds of $x_{t}, t \in T$. Let $\left(\phi_{t}\right)_{t \in T}$ be a unital field of positive linear mappings $\phi_{t}: \rightarrow \mathcal{B}$ from to another unital $C^{*}$ - algebra $\mathcal{B}$. If

$$
\left(m_{x}, M_{x}\right) \cap\left[m_{t}, M_{t}\right]=\varnothing, \quad t \in T
$$

where $m_{x}$ and $M_{x}, m_{x} \leq M_{x}$, are the bounds of the self-adjoint operator $x=\int_{T} \phi_{t}\left(x_{t}\right) d \mu(t)$, then

$$
f\left(\int_{T} \phi_{t}\left(x_{t}\right) d \mu(t)\right) \leq \int_{T} \phi_{t}\left(f\left(x_{t}\right)\right) d \mu(t)
$$

holds for every continuous convex function $f: I \rightarrow \mathbb{R}$ provided that the interval $I$ contains all $m_{t}, M_{t}$.

If $f: I \rightarrow \mathbb{R}$ is concave, then the reverse inequality is valid in ( $\square$ ).

We prove only the case when $f$ is a convex function. If we denote $m=\inf \left\{m_{t}\right\}$ and $M=\sup _{t \in T}\left\{M_{t}\right\}$, then $\quad[m, M] \subseteq I \quad$ and $\quad m 1_{H} \leq A_{t} \leq M 1_{H}, \quad t \in T$. It follows $m 1_{K} \leq \int_{T} \phi_{t}\left(x_{t}\right) d \mu(t) \leq M 1_{K}$. Therefore $\left[m_{x}, M_{x}\right] \subseteq[m, M] \subseteq I$.

a) Let $m_{x}<M_{x}$. Since $f$ is convex on $\left[m_{x}, M_{x}\right]$, then

$$
f(z) \leq \frac{M_{x}-z}{M_{x}-m_{x}} f\left(m_{x}\right)+\frac{z-m_{x}}{M_{x}-m_{x}} f\left(M_{x}\right), \quad z \in\left[m_{x}, M_{x}\right]
$$

but since $f$ is convex on $\left[m_{t}, M_{t}\right]$ and since $\left(m_{x}, M_{x}\right) \cap\left[m_{t}, M_{t}\right]=\varnothing$, then

$$
f(z) \geq \frac{M_{x}-z}{M_{x}-m_{x}} f\left(m_{x}\right)+\frac{z-m_{x}}{M_{x}-m_{x}} f\left(M_{x}\right), \quad z \in\left[m_{t}, M_{t}\right], \quad t \in T
$$


Since $m_{x} 1_{K} \leq \int_{T} \phi_{t}\left(x_{t}\right) d \mu(t) \leq M_{x} 1_{K}$, then by using functional calculus, it follows from ( $\square$ )

$$
f\left(\int_{T} \phi_{t}\left(x_{t}\right) d \mu(t)\right) \leq \frac{M_{x} 1_{K}-\int_{T} \phi_{t}\left(x_{t}\right) d \mu(t)}{M_{x}-m_{x}} f\left(m_{x}\right)+\frac{\int_{T} \phi_{t}\left(x_{t}\right) d \mu(t)-m_{x} 1_{K}}{M_{x}-m_{x}} f\left(M_{x}\right)
$$

On the other hand, since $m_{t} 1_{H} \leq x_{t} \leq M_{t} 1_{H}, t \in T$, then by using functional calculus, it follows from (口)

$$
f\left(x_{t}\right) \geq \frac{M_{x} 1_{H}-x_{t}}{M_{x}-m_{x}} f\left(m_{x}\right)+\frac{x_{t}-m_{x} 1_{H}}{M_{x}-m_{x}} f\left(M_{x}\right), \quad t \in T
$$

Applying a positive linear mapping $\phi_{t}$ and summing, we obtain

$$
\int_{T} \phi_{t}\left(f\left(x_{t}\right)\right) d \mu(t) \geq \frac{M_{x} 1_{K}-\int_{T} \phi_{t}\left(x_{t}\right) d \mu(t)}{M_{x}-m_{x}} f\left(m_{x}\right)+\frac{\int_{T} \phi_{t}\left(x_{t}\right) d \mu(t)-m_{x} 1_{K}}{M_{x}-m_{x}} f\left(M_{x}\right)
$$

since $\int_{T} \phi_{t}\left(1_{H}\right) d \mu(t)=1_{K}$. Combining the two inequalities ( $\left.\square\right)$ and ( $\left.\square\right)$, we have the desired inequality (口).

b) Let $m_{x}=M_{x}$. Since $f$ is convex on $[m, M]$, we have

$$
f(z) \geq f\left(m_{x}\right)+l\left(m_{x}\right)\left(z-m_{x}\right) \quad \text { for every } z \in[m, M]
$$

where $l$ is the subdifferential of $f$. Since $m 1_{H} \leq x_{t} \leq M 1_{H}, t \in T$, then by using functional calculus, applying a positive linear mapping $\phi_{t}$ and summing, we obtain from ( $\square$ )

$$
\int_{T} \phi_{t}\left(f\left(x_{t}\right)\right) d \mu(t) \geq f\left(m_{x}\right) 1_{K}+l\left(m_{x}\right)\left(\int_{T} \phi_{t}\left(x_{t}\right) d \mu(t)-m_{x} 1_{K}\right)
$$

Since $m_{x} 1_{K}=\int_{T} \phi_{t}\left(x_{t}\right) d \mu(t)$, it follows

$$
\int_{T} \phi_{t}\left(f\left(x_{t}\right)\right) d \mu(t) \geq f\left(m_{x}\right) 1_{K}=f\left(\int_{T} \phi_{t}\left(x_{t}\right) d \mu(t)\right)
$$

which is the desired inequality (ㅁ). Putting $\phi_{t}(y)=a_{t} y$ for every $y \in$, where $a_{t} \geq 0$ is a real number, we obtain the following obvious corollary of Theorem $\square$.

Corollary 7 Let $\left(x_{t}\right)_{t \in T}$ be a bounded continuous field of self-adjoint elements in a unital $C^{*}$ algebra defined on a locally compact Hausdorff space $T$ equipped with a bounded Radon measure $\mu$. Let $m_{t}$ and $M_{t}, m_{t} \leq M_{t}$, be the bounds of $x_{t}, t \in T$. Let $\left(a_{t}\right)_{t \in T}$ be a continuous field of nonnegative real numbers such that $\int_{T} a_{t} d \mu(t)=1$. If 


$$
\left(m_{x}, M_{x}\right) \cap\left[m_{t}, M_{t}\right]=\varnothing, \quad t \in T
$$

where $m_{x}$ and $M_{x}, m_{x} \leq M_{x}$, are the bounds of the self-adjoint operator $x=\int_{T} a_{t} x_{t} d \mu(t)$, then

$$
f\left(\int_{T} a_{t} x_{t} d \mu(t)\right) \leq \int_{T} a_{t} f\left(x_{t}\right) d \mu(t)
$$

holds for every continuous convex function $f: I \rightarrow \mathbb{R}$ provided that the interval $I$ contains all $m_{t}, M_{t}$.

\subsection{Converses of Jensen's inequality with conditions on spectra}

Using the condition on spectra we obtain the following extension of Theorem $\square$.

Theorem 8 Let $\left(x_{t}\right)_{t \in T}$ be a bounded continuous field of self-adjoint elements in a unital $C^{*}$ algebra defined on a locally compact Hausdorff space $T$ equipped with a bounded Radon measure $\mu$. Furthermore, let $\left(\phi_{t}\right)_{t \in T}$ be a field of positive linear mappings $\phi_{t}: \rightarrow \mathcal{B}$ from to another unital $C^{*}$-algebra $\mathcal{B}$, such that the function $t \mapsto \phi_{t}\left(1_{H}\right)$ is integrable with $\int_{T} \phi_{t}\left(1_{H}\right) d \mu(t)=k 1_{K}$ for some positive scalar $k$. Let $m_{t}$ and $M_{t}, m_{t} \leq M_{t}$, be the bounds of $x_{t}$, $t \in T, \quad m=\inf _{t \in T}\left\{m_{t}\right\}, \quad M=\sup _{t \in T}\left\{M_{t}\right\}$, and $m_{x}$ and $M_{x}, m_{x}<M_{x}$, be the bounds of $x=\int_{T} \phi_{t}\left(x_{t}\right) d \mu(t)$. If

$$
\left(m_{x}, M_{x}\right) \cap\left[m_{t}, M_{t}\right]=\varnothing, \quad t \in T
$$

and $f:[m, M] \rightarrow \mathbb{R}, g:\left[m_{x}, M_{x}\right] \rightarrow \mathbb{R}, \quad F: U \times V \rightarrow \mathbb{R}$ are functions such that $(k f)([m, M]) \subset U, g\left(\left[m_{x}, M_{x}\right]\right) \subset V, f$ is convex, $F$ is bounded and operator monotone in the first variable, then

$$
\begin{gathered}
\quad \inf _{m_{x} \leq z \leq M_{x}} F\left[\frac{M_{x} k-z}{M_{x}-m_{x}} f\left(m_{x}\right)+\frac{z-k m_{x}}{M_{x}-m_{x}} f\left(M_{x}\right), g(z)\right] 1_{K} \\
F\left[\int_{T} \phi_{t}\left(f\left(x_{t}\right)\right) d \mu(t), g\left(\int_{T} \phi_{t}\left(x_{t}\right) d \mu(t)\right)\right] \\
\leq \sup _{m_{x} \leq z \leq M_{x}} F\left[\frac{M k-z}{M-m} f(m)+\frac{z-k m}{M-m} f(M), g(z)\right] 1_{K}
\end{gathered}
$$

In the dual case (when $f$ is concave) the opposite inequalities hold in ( $\square$ ) by replacing inf and sup with sup and inf, respectively.

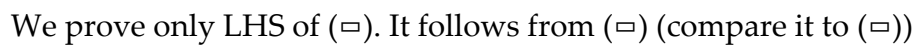




$$
\int_{T} \phi_{t}\left(f\left(x_{t}\right)\right) d \mu(t) \geq \frac{M_{x} k 1_{K}-\int_{T} \phi_{t}\left(x_{t}\right) d \mu(t)}{M_{x}-m_{x}} f\left(m_{x}\right)+\frac{\int_{T} \phi_{t}\left(x_{t}\right) d \mu(t)-m_{x} k 1_{K}}{M_{x}-m_{x}} f\left(M_{x}\right)
$$

since $\int_{T} \phi_{t}\left(1_{H}\right) d \mu(t)=k 1_{K}$. By using operator monotonicity of $F(\cdot, v)$ we obtain

$\left[\int_{T} \phi_{t}\left(f\left(x_{t}\right)\right) d \mu(t), g\left(\int_{T} \phi_{t}\left(x_{t}\right) d \mu(t)\right)\right] \geq F\left[\frac{M_{x} k 1_{K}-\int_{T} \phi_{t}\left(x_{t}\right) d \mu(t)}{M_{x}-m_{x}} f\left(m_{x}\right)+\frac{\int_{T} \phi_{t}\left(x_{t}\right) d \mu(t)-m_{x} k 1_{K}}{M_{x}-m_{x}} f\left(M_{x}\right), g\left(\int_{T} \phi\left(\gamma\left(x_{t}\right) d \mu(t)\right)\right]\right.$ $\operatorname{mxzMx} \mathrm{F}[M x$ k-zMx-mxf(mx)+z-kmxMx-mxf(Mx),g(z)] 1K

Putting $F(u, v)=u-\alpha v$ or $F(u, v)=v^{-1 / 2} u v^{-1 / 2}$ in Theorem $\square$, we obtain the next corollary.

Corollary 9 Let $\left(x_{t}\right)_{t \in T}, m_{t}, M_{t}, m_{x}, M_{x}, m, M,\left(\phi_{t}\right)_{t \in T}$ be as in Theorem $\square$ and $f:[m, M] \rightarrow \mathbb{R}, g:\left[m_{x}, M_{x}\right] \rightarrow \mathbb{R}$ be continuous functions. If

$$
\left(m_{x}, M_{x}\right) \cap\left[m_{t}, M_{t}\right]=\varnothing, \quad t \in T
$$

and $f$ is convex, then for any $\alpha \in \mathbb{R}$

$$
\begin{gathered}
\min _{m_{x} \leq z \leq M_{x}}\left\{\frac{M_{x} k-z}{M_{x}-m_{x}} f\left(m_{x}\right)+\frac{z-k m_{x}}{M_{x}-m_{x}} f\left(M_{x}\right)-g(z)\right\} 1_{K}+\alpha g\left(\int_{T} \phi_{t}\left(x_{t}\right) d \mu(t)\right) \\
\quad \leq \int_{T} \phi_{t}\left(f\left(x_{t}\right)\right) d \mu(t) \\
\leq \alpha g\left(\int_{T} \phi_{t}\left(x_{t}\right) d \mu(t)\right)+\max _{m_{x} \leq z \leq M_{x}}\left\{\frac{M k-z}{M-m} f(m)+\frac{z-k m}{M-m} f(M)-g(z)\right\} 1_{K}
\end{gathered}
$$

If additionally $g>0$ on $\left[m_{x}, M_{x}\right]$, then

$$
\begin{gathered}
\left\{\begin{array}{c}
\frac{M_{x} k-z}{M_{x}-m_{x}} f\left(m_{x}\right)+\frac{z-k m_{x}}{M_{x}-m_{x}} f\left(M_{x}\right) \\
\min _{m_{x} \leq z \leq M_{x}}(z)
\end{array}\right) g\left(\int_{T} \phi_{t}\left(x_{t}\right) d \mu(t)\right) \\
\leq \int_{T} \phi_{t}\left(f\left(x_{t}\right)\right) d \mu(t) \leq \max _{m_{x} \leq z \leq M_{x}}\left\{\frac{\frac{M k-z}{M-m} f(m)+\frac{z-k m}{M-m} f(M)}{g(z)}\right\}_{g}\left(\int_{T} \phi_{t}\left(x_{t}\right) d \mu(t)\right)
\end{gathered}
$$

In the dual case (when $f$ is concave) the opposite inequalities hold in ( $\square$ ) by replacing min and max with max and min, respectively. If additionally $g>0$ on $\left[m_{x}, M_{x}\right]$, then the oppo- 
site inequalities also hold in (־) by replacing min and max with max and min, respectively.

\section{Refined Jensen's inequality}

In this section we present a refinement of Jensen's inequality for real valued continuous convex functions given in Theorem $\square$. A discrete version of this result is given in [19].

To obtain our result we need the following two lemmas.

Lemma 10 Let $f$ be a convex function on an interval $I, m, M \in I$ and $p_{1}, p_{2} \in[0,1]$ such that $p_{1}+p_{2}=1$. Then

$$
\min \left\{p_{1}, p_{2}\right\}\left[f(m)+f(M)-2 f\left(\frac{m+M}{2}\right)\right] \leq p_{1} f(m)+p_{2} f(M)-f\left(p_{1} m+p_{2} M\right)
$$

These results follows from Theorem 1, p. 717[20].

Lemma 11 Let $x$ be a bounded self-adjoint elements in a unital $C^{*}$-algebra of operators on some Hilbert space $H$. If the spectrum of $x$ is in $[m, M]$, for some scalars $m<M$, then

$$
\begin{aligned}
f(x) & \leq \frac{M 1_{H}-x}{M-m} f(m)+\frac{x-m 1_{H}}{M-m} f(M)-\delta_{f} \tilde{x} \\
\text { (resp. } f(x) & \geq \frac{M 1_{H}-x}{M-m} f(m)+\frac{x-m 1_{H}}{M-m} f(M)+\delta_{f} \tilde{x}
\end{aligned}
$$

holds for every continuous convex (resp. concave) function $f:[m, M] \rightarrow \mathbb{R}$, where

$$
\begin{gathered}
\delta_{f}=f(m)+f(M)-2 f\left(\frac{m+M}{2}\right) \quad\left(\text { resp. } \delta_{f}=2 f\left(\frac{m+M}{2}\right)-f(m)-f(M)\right) \\
\text { and } \tilde{x}=\frac{1}{2} 1_{H}-\frac{1}{M-m}\left|x-\frac{m+M}{2} 1_{H}\right|
\end{gathered}
$$

We prove only the convex case. It follows from (ㅁ) that

$$
\begin{aligned}
f\left(p_{1} m+p_{2} M\right) & \leq p_{1} f(m)+p_{2} f(M) \\
& -\min \left\{p_{1}, p_{2}\right\}\left(f(m)+f(M)-2 f\left(\frac{m+M}{2}\right)\right)
\end{aligned}
$$

for every $p_{1}, p_{2} \in[0,1]$ such that $p_{1}+p_{2}=1$. For any $z \in[m, M]$ we can write 


$$
f(z)=f\left(\frac{M-z}{M-m} m+\frac{z-m}{M-m} M\right)
$$

Then by using (ㅁ) for $p_{1}=\frac{M-z}{M-m}$ and $p_{2}=\frac{z-m}{M-m}$ we obtain

$$
\begin{aligned}
f(z) & \leq \frac{M-z}{M-m} f(m)+\frac{z-m}{M-m} f(M) \\
& -\left(\frac{1}{2}-\frac{1}{M-m}\left|z-\frac{m+M}{2}\right|\right)\left(f(m)+f(M)-2 f\left(\frac{m+M}{2}\right)\right)
\end{aligned}
$$

since

$$
\min \left\{\frac{M-z}{M-m}, \frac{z-m}{M-m}\right\}=\frac{1}{2}-\frac{1}{M-m}\left|z-\frac{m+M}{2}\right|
$$

Finally we use the continuous functional calculus for a self-adjoint operator $x$ : $f, g \in(I), S p(x) \subseteq I$ and $f \leq g$ on $I$ implies $f(x) \leq g(x)$; and $h(z)=|z|$ implies $h(x)=|x|$. Then by using (口) we obtain the desired inequality (口).

Theorem 12 Let $\left(x_{t}\right)_{t \in T}$ be a bounded continuous field of self-adjoint elements in a unital $C^{*}$-algebra defined on a locally compact Hausdorff space $T$ equipped with a bounded Radon measure $\mu$. Let $m_{t}$ and $M_{t}, m_{t} \leq M_{t}$, be the bounds of $x_{t}, t \in T$. Let $\left(\phi_{t}\right)_{t \in T}$ be a unital field of positive linear mappings $\phi_{t}: \rightarrow \mathcal{B}$ from to another unital $C^{*}$ - algebra $\mathcal{B}$. Let

$$
\left(m_{x}, M_{x}\right) \cap\left[m_{t}, M_{t}\right]=\varnothing, \quad t \in T, \quad \text { and } \quad m<M
$$

where $m_{x}$ and $M_{x}, m_{x} \leq M_{x}$, be the bounds of the operator $x=\int_{T} \phi_{t}\left(x_{t}\right) d \mu(t)$ and

$$
m=\sup \left\{M_{t}: M_{t} \leq m_{x}, t \in T\right\}, M=\inf \left\{m_{t}: m_{t} \geq M_{x}, t \in T\right\}
$$

If $f: I \rightarrow \mathbb{R}$ is a continuous convex (resp. concave) function provided that the interval $I$ contains all $m_{t}, M_{t}$, then

$$
f\left(\int_{T} \phi_{t}\left(x_{t}\right) d \mu(t)\right) \leq \int_{T} \phi_{t}\left(f\left(x_{t}\right)\right) d \mu(t)-\delta_{f} \tilde{x} \leq \int_{T} \phi_{t}\left(f\left(x_{t}\right)\right) d \mu(t)
$$

(resp.

$$
\left.f\left(\int_{T} \phi_{t}\left(x_{t}\right) d \mu(t)\right) \geq \int_{T} \phi_{t}\left(f\left(x_{t}\right)\right) d \mu(t)-\delta_{f} \tilde{x} \geq \int_{T} \phi_{t}\left(f\left(x_{t}\right)\right) d \mu(t)\right)
$$

holds, where 


$$
\begin{aligned}
\delta_{f} & \equiv \delta_{f}(\bar{m}, \bar{M})=f(\bar{m})+f(\bar{M})-2 f\left(\frac{\bar{m}+\bar{M}}{2}\right) \\
\text { (resp. } \quad \delta_{f} \equiv \delta_{f}(\bar{m}, \bar{M}) & =2 f\left(\frac{\bar{m}+\bar{M}}{2}\right)-f(\bar{m})-f(\bar{M}) \mid \\
\tilde{x} & \equiv \tilde{x}_{x}(\bar{m}, \bar{M})=\frac{1}{2} 1_{K}-\frac{1}{M-\bar{m}}\left|x-\frac{\bar{m}+\bar{M}}{2} 1_{K}\right|
\end{aligned}
$$

and $\bar{m} \in\left[m, m_{A}\right], \bar{M} \in\left[M_{A}, M\right], \bar{m}<\bar{M}$, are arbitrary numbers.

We prove only the convex case. Since $x=\int_{T} \phi_{t}\left(x_{t}\right) d \mu(t) \in \mathcal{B}$ is the self-adjoint elements such that $\bar{m} 1_{K} \leq m_{x} 1_{K} \leq \int_{T} \phi_{t}\left(x_{t}\right) d \mu(t) \leq M_{x} 1_{K} \leq \bar{M} 1_{K}$ and $f$ is convex on $[\bar{m}, \bar{M}] \subseteq I$, then by Lemma $\square$ we obtain

$$
f\left(\int_{T} \phi_{t}\left(x_{t}\right) d \mu(t)\right) \leq \frac{\bar{M} 1_{K}-\int_{T} \phi_{t}\left(x_{t}\right) d \mu(t)}{\bar{M}-\bar{m}} f(\bar{m})+\frac{\int_{T} \phi_{t}\left(x_{t}\right) d \mu(t)-\bar{m} 1_{K}}{\bar{M}-\bar{m}} f(\bar{M})-\delta_{f} \tilde{x}
$$

where $\delta_{f}$ and $\tilde{x}$ are defined by (ㅁ).

But since $f$ is convex on $\left[m_{t}, M_{t}\right]$ and $\left(m_{x}, M_{x}\right) \cap\left[m_{t}, M_{t}\right]=\varnothing$ implies $(\bar{m}, \bar{M}) \cap\left[m_{t}, M_{t}\right]=\varnothing$, then

$$
f\left(x_{t}\right) \geq \frac{\bar{M} 1_{H}-x_{t}}{\bar{M}-\bar{m}} f(\bar{m})+\frac{x_{t}-\bar{m} 1_{H}}{\bar{M}-\bar{m}} f(\bar{M}), \quad t \in T
$$

Applying a positive linear mapping $\phi_{t}$, integrating and adding $-\delta_{f} \tilde{x}$, we obtain

$$
\int_{T} \phi_{t}\left(f\left(x_{t}\right)\right) d \mu(t)-\delta_{f} \tilde{x} \geq \frac{\bar{M} 1_{K}-\int_{T} \phi_{t}\left(x_{t}\right) d \mu(t)}{\bar{M}-\bar{m}} f(\bar{m})+\frac{\int_{T} \phi_{t}\left(x_{t}\right) d \mu(t)-\bar{m} 1_{K}}{\bar{M}-\bar{m}} f(\bar{M})-\delta_{f} \tilde{x}
$$

since $\int_{T} \phi_{t}\left(1_{H}\right) d \mu(t)=1_{K}$. Combining the two inequalities ( $\left.\square\right)$ and ( $\left.\square\right)$, we have LHS of ( $\left.\square\right)$. Since $\delta_{f} \geq 0$ and $\tilde{x} \geq 0$, then we have RHS of $(\square)$.

If $m<M$ and $m_{x}=M_{x}$, then the inequality ( $\left.\square\right)$ holds, but $\delta_{f}\left(m_{x}, M_{x}\right) \tilde{x}\left(m_{x}, M_{x}\right)$ is not defined (see Example $\square$ I) and II)).

Example 13 We give examples for the matrix cases and $T=\{1,2\}$. Then we have refined inequalities given in Fig. 2.

We put $f(t)=t^{4}$ which is convex but not operator convex in (口). Also, we define mappings $\Phi_{1}, \Phi_{2}: M_{3}(\mathbb{C}) \rightarrow M_{2}(\mathbb{C}) \quad$ as $\quad$ follows: $\quad \Phi_{1}\left(\left(a_{i j}\right)_{1 \leq i, j \leq 3}\right)=\frac{1}{2}\left(a_{i j}\right)_{1 \leq i, j \leq 2^{\prime}} \quad \Phi_{2}=\Phi_{1} \quad$ (then $\left.\Phi_{1}\left(I_{3}\right)+\Phi_{2}\left(I_{3}\right)=I_{2}\right)$. 


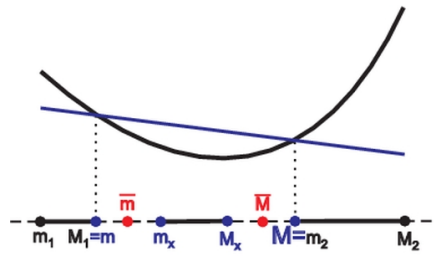

$f\left(\phi_{1}\left(x_{1}\right)\right)+f\left(\phi_{2}\left(x_{2}\right)\right) \leq \phi_{1}\left(f\left(x_{1}\right)\right)+\phi_{2}\left(f\left(x_{2}\right)\right)-\delta_{1} \tilde{x}$,

where

$\delta_{\mathrm{f}}=\mathrm{f}(\overline{\mathrm{m}})+\mathrm{f}(\overline{\mathrm{M}})-2 \mathrm{f}((\overline{\mathrm{M}}+\overline{\mathrm{m}}) / 2)$,

$\tilde{\mathrm{x}}=\frac{1}{2} 1_{\kappa}-\frac{1}{\overline{\mathrm{M}}-\overline{\mathrm{m}}}\left|\phi_{1}\left(\mathrm{x}_{1}\right)+\phi_{2}\left(\mathrm{x}_{2}\right)-\frac{\overline{\mathrm{M}}+\overline{\mathrm{m}}}{2} 1_{\kappa}\right|$

Figure 2. Refinement for two operators and a convex function $f$

I) First, we observe an example when $\delta_{f} \widetilde{X}$ is equal to the difference RHS and LHS of Jensen's inequality. If $X_{1}=-3 I_{3}$ and $X_{2}=2 I_{3}$, then $X=\Phi_{1}\left(X_{1}\right)+\Phi_{2}\left(X_{2}\right)=-0.5 I_{2}$, so $m=-3$, $M=2$. We also put $\bar{m}=-3$ and $\bar{M}=2$. We obtain

$$
\left(\Phi_{1}\left(X_{1}\right)+\Phi_{2}\left(X_{2}\right)\right)^{4}=0.0625 I_{2}<48.5 I_{2}=\Phi_{1}\left(X_{1}^{4}\right)+\Phi_{2}\left(X_{2}^{4}\right)
$$

and its improvement

$$
\left(\Phi_{1}\left(X_{1}\right)+\Phi_{2}\left(X_{2}\right)\right)^{4}=0.0625 I_{2}=\Phi_{1}\left(X_{1}^{4}\right)+\Phi_{2}\left(X_{2}^{4}\right)-48.4375 I_{2}
$$

since $\delta_{f}=96.875, \widetilde{X}=0.5 I_{2}$. We remark that in this case $m_{x}=M_{x}=-1 / 2$ and $\widetilde{X}\left(m_{x}, M_{x}\right)$ is not defined.

II) Next, we observe an example when $\delta_{f} \widetilde{X}$ is not equal to the difference RHS and LHS of Jensen's inequality and $m_{x}=M_{x}$. If

$$
X_{1}=\left(\begin{array}{ccc}
-1 & 0 & 0 \\
0 & -2 & 0 \\
0 & 0 & -1
\end{array}\right), \quad X_{2}=\left(\begin{array}{lll}
2 & 0 & 0 \\
0 & 3 & 0 \\
0 & 0 & 4
\end{array}\right) \text {, then } X=\frac{1}{2}\left(\begin{array}{ll}
1 & 0 \\
0 & 1
\end{array}\right) \text { and } m=-1, \quad M=2
$$

In this case $\tilde{x}\left(m_{x}, M_{x}\right)$ is not defined, since $m_{x}=M_{x}=1 / 2$. We have

$$
\left(\Phi_{1}\left(X_{1}\right)+\Phi_{2}\left(X_{2}\right)\right)^{4}=\frac{1}{16}\left(\begin{array}{ll}
1 & 0 \\
0 & 1
\end{array}\right)<\left(\begin{array}{cc}
\frac{17}{2} & 0 \\
0 & \frac{97}{2}
\end{array}\right)=\Phi_{1}\left(X_{1}^{4}\right)+\Phi_{2}\left(X_{2}^{4}\right)
$$

and putting $\bar{m}=-1, \bar{M}=2$ we obtain $\delta_{f}=135 / 8, \widetilde{X}=I_{2} / 2$ which give the following improvement

$$
\left(\Phi_{1}\left(X_{1}\right)+\Phi_{2}\left(X_{2}\right)\right)^{4}=\frac{1}{16}\left(\begin{array}{ll}
1 & 0 \\
0 & 1
\end{array}\right)<\frac{1}{16}\left(\begin{array}{cc}
1 & 0 \\
0 & 641
\end{array}\right)=\Phi_{1}\left(X_{1}^{4}\right)+\Phi_{2}\left(X_{2}^{4}\right)-\frac{135}{16}\left(\begin{array}{ll}
1 & 0 \\
0 & 1
\end{array}\right)
$$


III) Next, we observe an example with matrices that are not special. If

$$
X_{1}=\left(\begin{array}{ccc}
-4 & 1 & 1 \\
1 & -2 & -1 \\
1 & -1 & -1
\end{array}\right) \text { and } X_{2}=\left(\begin{array}{ccc}
5 & -1 & -1 \\
-1 & 2 & 1 \\
-1 & 1 & 3
\end{array}\right), \quad \text { then } X=\frac{1}{2}\left(\begin{array}{ll}
1 & 0 \\
0 & 0
\end{array}\right)
$$

so $m_{1}=-4.8662, M_{1}=-0.3446, m_{2}=1.3446, M_{2}=5.8662, m=-0.3446, M=1.3446$ and we put $\bar{m}=m, \bar{M}=M$ (rounded to four decimal places). We have

$$
\left(\Phi_{1}\left(X_{1}\right)+\Phi_{2}\left(X_{2}\right)\right)^{4}=\frac{1}{16}\left(\begin{array}{ll}
1 & 0 \\
0 & 0
\end{array}\right)<\left(\begin{array}{cc}
\frac{1283}{2} & -255 \\
-255 & \frac{237}{2}
\end{array}\right)=\Phi_{1}\left(X_{1}^{4}\right)+\Phi_{2}\left(X_{2}^{4}\right)
$$

and its improvement

$$
\begin{aligned}
& \left(\Phi_{1}\left(X_{1}\right)+\Phi_{2}\left(X_{2}\right)\right)^{4}=\frac{1}{16}\left(\begin{array}{ll}
1 & 0 \\
0 & 0
\end{array}\right) \\
< & \left(\begin{array}{cc}
639.9213 & -255 \\
-255 & 117.8559
\end{array}\right)=\Phi_{1}\left(X_{1}^{4}\right)+\Phi_{2}\left(X_{2}^{4}\right)-\left(\begin{array}{cc}
1.5787 & 0 \\
0 & 0.6441
\end{array}\right)
\end{aligned}
$$

(rounded to four decimal places), since $\delta_{f}=3.1574, \widetilde{X}=\left(\begin{array}{cc}0.5 & 0 \\ 0 & 0.2040\end{array}\right)$. But, if we put $\bar{m}=m_{x}=0, \bar{M}=M_{x}=0.5$, then $\widetilde{X}=\mathbf{0}$, so we do not have an improvement of Jensen's inequality. Also, if we put $\bar{m}=0, \bar{M}=1$, then $\widetilde{X}=0.5\left(\begin{array}{ll}1 & 0 \\ 0 & 1\end{array}\right), \delta_{f}=7 / 8$ and $\delta_{f} \widetilde{X}=0.4375\left(\begin{array}{ll}1 & 0 \\ 0 & 1\end{array}\right)$, which is worse than the above improvement.

Putting $\Phi_{t}(y)=a_{t} y$ for every $y \in$, where $a_{t} \geq 0$ is a real number, we obtain the following obvious corollary of Theorem $\square$.

Corollary 14 Let $\left(x_{t}\right)_{t \in T}$ be a bounded continuous field of self-adjoint elements in a unital $C^{*}$-algebra defined on a locally compact Hausdorff space $T$ equipped with a bounded Radon measure $\mu$. Let $m_{t}$ and $M_{t}, m_{t} \leq M_{t}$, be the bounds of $x_{t}, t \in T$. Let $\left(a_{t}\right)_{t \in T}$ be a continuous field of nonnegative real numbers such that $\int_{T} a_{t} d \mu(t)=1$. Let

$$
\left(m_{x}, M_{x}\right) \cap\left[m_{t}, M_{t}\right]=\varnothing, \quad t \in T, \quad \text { and } \quad m<M
$$

where $m_{x}$ and $M_{x}, m_{x} \leq M_{x}$, are the bounds of the operator $x=\int_{T} \phi_{t}\left(x_{t}\right) d \mu(t)$ and

$$
m=\sup \left\{M_{t}: M_{t} \leq m_{x}, t \in T\right\}, M=\inf \left\{m_{t}: m_{t} \geq M_{x^{\prime}}, t \in T\right\}
$$


If $f: I \rightarrow \mathbb{R}$ is a continuous convex (resp. concave) function provided that the interval $I$ contains all $m_{t}, M_{t}$, then

$$
\begin{aligned}
f\left(\int_{T} a_{t} x_{t} d \mu(t)\right) & \leq \int_{T} a_{t} f\left(x_{t}\right) d \mu(t)-\delta_{f} \tilde{\tilde{x}} \leq \int_{T} a_{t} f\left(x_{t}\right) d \mu(t) \\
\text { (resp. } f\left(\int_{T} a_{t} x_{t} d \mu(t)\right) & \left.\geq \int_{T} a_{t} f\left(x_{t}\right) d \mu(t)+\delta_{f} \tilde{\tilde{x}} \geq \int_{T} a_{t} f\left(x_{t}\right) d \mu(t)\right)
\end{aligned}
$$

holds, where $\delta_{f}$ is defined by (口), $\tilde{\tilde{x}}=\frac{1}{2} 1_{H}-\frac{1}{M-\bar{m}}\left|\int_{T} a_{t} x_{t} d \mu(t)-\frac{\bar{m}+\bar{M}}{2} 1_{H}\right|$ and $\bar{m} \in\left[m, m_{A}\right]$, $\bar{M} \in\left[M_{A}, M\right], \bar{m}<\bar{M}$, are arbitrary numbers.

\section{Extension Jensen's inequality}

In this section we present an extension of Jensen's operator inequality for $n$ - tuples of selfadjoint operators, unital $n$ - tuples of positive linear mappings and real valued continuous convex functions with conditions on the spectra of the operators.

In a discrete version of Theorem $\square$ we prove that Jensen's operator inequality holds for every continuous convex function and for every $n$ - tuple of self-adjoint operators $\left(A_{1}, \ldots, A_{n}\right)$, for every $n$ - tuple of positive linear mappings $\left(\Phi_{1}, \ldots, \Phi_{n}\right)$ in the case when the interval with bounds of the operator $A=\sum_{i=1}^{n} \Phi_{i}\left(A_{i}\right)$ has no intersection points with the interval with bounds of the operator $A_{i}$ for each $i=1, \ldots, n$, i.e. when $\left(m_{A}, M_{A}\right) \cap\left[m_{i}, M_{i}\right]=\varnothing$ for $i=1, \ldots, n$, where $m_{A}$ and $M_{A}, m_{A} \leq M_{A}$, are the bounds of $A$, and $m_{i}$ and $M_{i}, m_{i} \leq M_{i}$, are the bounds of $A_{i}, i=1, \ldots, n$. It is interesting to consider the case when $\left(m_{A}, M_{A}\right) \cap\left[m_{i}, M_{i}\right]=\varnothing$ is valid for several $i \in\{1, \ldots, n\}$, but not for all $i=1, \ldots, n$. We study it in the following theorem (see [21]).

Theorem 15 Let $\left(A_{1}, \ldots, A_{n}\right)$ be an $n$-tuple of self-adjoint operators $A_{i} \in B(H)$ with the bounds $m_{i}$ and $M_{i}, m_{i} \leq M_{i}, i=1, \ldots, n$. Let $\left(\Phi_{1}, \ldots, \Phi_{n}\right)$ be an $n$ - tuple of positive linear mappings $\Phi_{i}: B(H) \rightarrow B(K)$, such that $\sum_{i=1}^{n} \Phi_{i}\left(1_{H}\right)=1_{K}$. For $1 \leq n_{1}<n$, we denote $m=\min \left\{m_{1}, \ldots, m_{n_{1}}\right\}, \quad M=\max \left\{M_{1}, \ldots, M_{n_{1}}\right\}$ and $\sum_{i=1}^{n_{1}} \Phi_{i}\left(1_{H}\right)=\alpha 1_{K}, \sum_{i=n_{1}+1}^{n} \Phi_{i}\left(1_{H}\right)=\beta 1_{K}$, where $\alpha, \beta>0, \alpha+\beta=1$. If

$$
(m, M) \cap\left[m_{i}, M_{i}\right]=\varnothing, \quad i=n_{1}+1, \ldots, n
$$

and one of two equalities

$$
\frac{1}{\alpha} \sum_{i=1}^{n_{1}} \Phi_{i}\left(A_{i}\right)=\frac{1}{\beta} \sum_{i=n_{1}+1}^{n} \Phi_{i}\left(A_{i}\right)=\sum_{i=1}^{n} \Phi_{i}\left(A_{i}\right)
$$


is valid, then

$$
\frac{1}{\alpha} \sum_{i=1}^{n_{1}} \Phi_{i}\left(f\left(A_{i}\right)\right) \leq \sum_{i=1}^{n} \Phi_{i}\left(f\left(A_{i}\right)\right) \leq \frac{1}{\beta} \sum_{i=n_{1}+1}^{n} \Phi_{i}\left(f\left(A_{i}\right)\right)
$$

holds for every continuous convex function $f: I \rightarrow \mathbb{R}$ provided that the interval $I$ contains all $m_{i}, M_{i}, i=1, \ldots, n$. If $f: I \rightarrow \mathbb{R}$ is concave, then the reverse inequality is valid in ( $\square$ ).

We prove only the case when $f$ is a convex function. Let us denote

$$
A=\frac{1}{\alpha} \sum_{i=1}^{n_{1}} \Phi_{i}\left(A_{i}\right), \quad B=\frac{1}{\beta} \sum_{i=n_{1}+1}^{n} \Phi_{i}\left(A_{i}\right), \quad C=\sum_{i=1}^{n} \Phi_{i}\left(A_{i}\right)
$$

It is easy to verify that $A=B$ or $B=C$ or $A=C$ implies $A=B=C$.

a) Let $m<M$. Since $f$ is convex on $[m, M]$ and $\left[m_{i}, M_{i}\right] \subseteq[m, M]$ for $i=1, \ldots, n_{1}$, then

$$
f(z) \leq \frac{M-z}{M-m} f(m)+\frac{z-m}{M-m} f(M), \quad z \in\left[m_{i}, M_{i}\right] \text { for } i=1, \ldots, n_{1}
$$

but since $f$ is convex on all $\left[m_{i}, M_{i}\right]$ and $(m, M) \cap\left[m_{i}, M_{i}\right]=\varnothing$ for $i=n_{1}+1, \ldots, n$, then

$$
f(z) \geq \frac{M-z}{M-m} f(m)+\frac{z-m}{M-m} f(M), \quad z \in\left[m_{i}, M_{i}\right] \text { for } i=n_{1}+1, \ldots, n
$$

Since $m_{i} 1_{H} \leq A_{i} \leq M_{i} 1_{H}, i=1, \ldots, n_{1}$, it follows from (口)

$$
f\left(A_{i}\right) \leq \frac{M 1_{H}-A_{i}}{M-m} f(m)+\frac{A_{i}-m 1_{H}}{M-m} f(M), \quad i=1, \ldots, n_{1}
$$

Applying a positive linear mapping $\Phi_{i}$ and summing, we obtain

$$
\sum_{i=1}^{n_{1}} \Phi_{i}\left(f\left(A_{i}\right)\right) \leq \frac{M \alpha 1_{K}-\sum_{i=1}^{n_{1}} \Phi_{i}\left(A_{i}\right)}{M-m} f(m)+\frac{\sum_{i=1}^{n_{1}} \Phi_{i}\left(A_{i}\right)-m \alpha 1_{K}}{M-m} f(M)
$$

since $\sum_{i=1}^{n_{1}} \Phi_{i}\left(1_{H}\right)=\alpha 1_{K}$. It follows

$$
\frac{1}{\alpha} \sum_{i=1}^{n_{1}} \Phi_{i}\left(f\left(A_{i}\right)\right) \leq \frac{M 1_{K}-A}{M-m} f(m)+\frac{A-m 1_{K}}{M-m} f(M)
$$

Similarly to (口) in the case $m_{i} 1_{H} \leq A_{i} \leq M_{i} 1_{H}, i=n_{1}+1, \ldots, n$, it follows from (ㅁ) 


$$
\frac{1}{\beta} \sum_{i=n_{1}+1}^{n} \Phi_{i}\left(f\left(A_{i}\right)\right) \geq \frac{M 1_{K}-B}{M-m} f(m)+\frac{B-m 1_{K}}{M-m} f(M)
$$

Combining (ㅁ) and (ㅁ) and taking into account that $A=B$, we obtain

$$
\frac{1}{\alpha} \sum_{i=1}^{n_{1}} \Phi_{i}\left(f\left(A_{i}\right)\right) \leq \frac{1}{\beta} \sum_{i=n_{1}+1}^{n} \Phi_{i}\left(f\left(A_{i}\right)\right)
$$

It follows

$$
\begin{aligned}
\frac{1}{\alpha} \sum_{i=1}^{n_{1}} \Phi_{i}\left(f\left(A_{i}\right)\right) & =\sum_{i=1}^{n_{1}} \Phi_{i}\left(f\left(A_{i}\right)\right)+\frac{\beta}{\alpha} \sum_{i=1}^{n_{1}} \Phi_{i}\left(f\left(A_{i}\right)\right) & & \text { (by } \alpha+\beta=1) \\
& \leq \sum_{i=1}^{n_{1}} \Phi_{i}\left(f\left(A_{i}\right)\right)+\sum_{i=n_{1}+1}^{n} \Phi_{i}\left(f\left(A_{i}\right)\right) & & \text { (by }()) \\
& =\sum_{i=1}^{n} \Phi_{i}\left(f\left(A_{i}\right)\right) & & \text { (by }()) \\
& \leq \frac{\alpha}{\beta} \sum_{i=n_{1}+1}^{n} \Phi_{i}\left(f\left(A_{i}\right)\right)+\sum_{i=n_{1}+1}^{n} \Phi_{i}\left(f\left(A_{i}\right)\right) & & \text { (by } \alpha+\beta=1) \\
& =\frac{1}{\beta} \sum_{i=n_{1}+1}^{n} \Phi_{i}\left(f\left(A_{i}\right)\right) & &
\end{aligned}
$$

which gives the desired double inequality (口).

b) Let $m=M$. Since $\left[m_{i}, M_{i}\right] \subseteq[m, M]$ for $i=1, \ldots, n_{1}$, then $A_{i}=m 1_{H}$ and $f\left(A_{i}\right)=f(m) 1_{H}$ for $i=1, \ldots, n_{1}$. It follows

$$
\frac{1}{\alpha} \sum_{i=1}^{n_{1}} \Phi_{i}\left(A_{i}\right)=m 1_{K} \quad \text { and } \quad \frac{1}{\alpha} \sum_{i=1}^{n_{1}} \Phi_{i}\left(f\left(A_{i}\right)\right)=f(m) 1_{K}
$$

On the other hand, since $f$ is convex on $I$, we have

$$
f(z) \geq f(m)+l(m)(z-m) \quad \text { for every } z \in I
$$

where $l$ is the subdifferential of $f$. Replacing $z$ by $A_{i}$ for $i=n_{1}+1, \ldots, n$, applying $\Phi_{i}$ and summing, we obtain from (ㅁ) and (口)

$$
\begin{aligned}
\frac{1}{\beta} \sum_{i=n_{1}+1}^{n} \Phi_{i}\left(f\left(A_{i}\right)\right) & \geq f(m) 1_{K}+l(m)\left(\frac{1}{\beta} \sum_{i=n_{1}+1}^{n} \Phi_{i}\left(A_{i}\right)-m 1_{K}\right) \\
& =f(m) 1_{K}=\frac{1}{\alpha} \sum_{i=1}^{n_{1}} \Phi_{i}\left(f\left(A_{i}\right)\right)
\end{aligned}
$$


So (ㅁ) holds again. The remaining part of the proof is the same as in the case a).

Remark 16 We obtain the equivalent inequality to the one in Theorem $\square$ in the case when $\sum_{i=1}^{n} \Phi_{i}\left(1_{H}\right)=\gamma 1_{K}$, for some positive scalar $\gamma$. If $\alpha+\beta=\gamma$ and one of two equalities

$$
\frac{1}{\alpha} \sum_{i=1}^{n_{1}} \Phi_{i}\left(A_{i}\right)=\frac{1}{\beta} \sum_{i=n_{1}+1}^{n} \Phi_{i}\left(A_{i}\right)=\frac{1}{\gamma} \sum_{i=1}^{n} \Phi_{i}\left(A_{i}\right)
$$

is valid, then

$$
\frac{1}{\alpha} \sum_{i=1}^{n_{1}} \Phi_{i}\left(f\left(A_{i}\right)\right) \leq \frac{1}{\gamma} \sum_{i=1}^{n} \Phi_{i}\left(f\left(A_{i}\right)\right) \leq \frac{1}{\beta} \sum_{i=n_{1}+1}^{n} \Phi_{i}\left(f\left(A_{i}\right)\right)
$$

holds for every continuous convex function $f$.

Remark 17 Let the assumptions of Theorem $\square$ be valid.

1. We observe that the following inequality

$$
f\left(\frac{1}{\beta} \sum_{i=n_{1}+1}^{n} \Phi_{i}\left(A_{i}\right)\right) \leq \frac{1}{\beta} \sum_{i=n_{1}+1}^{n} \Phi_{i}\left(f\left(A_{i}\right)\right)
$$

holds for every continuous convex function $f: I \rightarrow \mathbb{R}$.

Indeed, by the assumptions of Theorem $\square$ we have

$$
m \alpha 1_{H} \leq \sum_{i=1}^{n_{1}} \Phi_{i}\left(f\left(A_{i}\right)\right) \leq M \alpha 1_{H} \quad \text { and } \quad \frac{1}{\alpha} \sum_{i=1}^{n_{1}} \Phi_{i}\left(A_{i}\right)=\frac{1}{\beta} \sum_{i=n_{1}+1}^{n} \Phi_{i}\left(A_{i}\right)
$$

which implies

$$
m 1_{H} \leq \sum_{i=n_{1}+1}^{n} \frac{1}{\beta} \Phi_{i}\left(f\left(A_{i}\right)\right) \leq M 1_{H}
$$

Also $(m, M) \cap\left[m_{i}, M_{i}\right]=\varnothing$ for $i=n_{1}+1, \ldots, n$ and $\sum_{i=n_{1}+1}^{n} \frac{1}{\beta} \Phi_{i}\left(1_{H}\right)=1_{K}$ hold. So we can apply Theorem $\square$ on operators $A_{n_{1}+1}, \ldots, A_{n}$ and mappings $\frac{1}{\beta} \Phi_{i}$ and obtain the desired inequality.

2. We denote by $m_{C}$ and $M_{C}$ the bounds of $C=\sum_{i=1}^{n} \Phi_{i}\left(A_{i}\right)$. If $\left(m_{C}, M_{C}\right) \cap\left[m_{i}, M_{i}\right]=\varnothing$, $i=1, \ldots, n_{1}$ or $f$ is an operator convex function on $[m, M]$, then the double inequality $(\square)$ can be extended from the left side if we use Jensen's operator inequality (see Theorem 2.1[16]) 


$$
\begin{aligned}
f\left(\sum_{i=1}^{n} \Phi_{i}\left(A_{i}\right)\right) & =f\left(\frac{1}{\alpha} \sum_{i=1}^{n_{1}} \Phi_{i}\left(A_{i}\right)\right) \\
& \leq \frac{1}{\alpha} \sum_{i=1}^{n_{1}} \Phi_{i}\left(f\left(A_{i}\right)\right) \leq \sum_{i=1}^{n} \Phi_{i}\left(f\left(A_{i}\right)\right) \leq \frac{1}{\beta} \sum_{i=n_{1}+1}^{n} \Phi_{i}\left(f\left(A_{i}\right)\right)
\end{aligned}
$$

Example 18 If neither assumptions $\left(m_{C}, M_{C}\right) \cap\left[m_{i}, M_{i}\right]=\varnothing, i=1, \ldots, n_{1}$, nor $f$ is operator convex in Remark $\square-2$. is satisfied and if $1<n_{1}<n$, then ( $\square$ ) can not be extended by Jensen's operator inequality, since it is not valid. Indeed, for $n_{1}=2$ we define mappings $\Phi_{1}, \Phi_{2}: M_{3}(\mathbb{C}) \rightarrow M_{2}(\mathbb{C})$ by $\Phi_{1}\left(\left(a_{i j}\right)_{1 \leq i, j \leq 3}\right)=\frac{\alpha}{2}\left(a_{i j}\right)_{1 \leq i, j \leq 2^{\prime}} \Phi_{2}=\Phi_{1}$. Then $\Phi_{1}\left(I_{3}\right)+\Phi_{2}\left(I_{3}\right)=\alpha I_{2}$. If

$$
A_{1}=2\left(\begin{array}{lll}
1 & 0 & 1 \\
0 & 0 & 1 \\
1 & 1 & 1
\end{array}\right) \text { and } A_{2}=2\left(\begin{array}{lll}
1 & 0 & 0 \\
0 & 0 & 0 \\
0 & 0 & 0
\end{array}\right)
$$

then

$$
\left(\frac{1}{\alpha} \Phi_{1}\left(A_{1}\right)+\frac{1}{\alpha} \Phi_{2}\left(A_{2}\right)\right)^{4}=\frac{1}{\alpha^{4}}\left(\begin{array}{cc}
16 & 0 \\
0 & 0
\end{array}\right) \neg \leq \frac{1}{\alpha}\left(\begin{array}{cc}
80 & 40 \\
40 & 24
\end{array}\right)=\frac{1}{\alpha} \Phi_{1}\left(A_{1}^{4}\right)+\frac{1}{\alpha} \Phi_{2}\left(A_{2}^{4}\right)
$$

for every $\alpha \in(0,1)$. We observe that $f(t)=t^{4}$ is not operator convex and $\left(m_{C}, M_{C}\right) \cap\left[m_{i}, M_{i}\right] \neq \varnothing$, since $C=A=\frac{1}{\alpha} \Phi_{1}\left(A_{1}\right)+\frac{1}{\alpha} \Phi_{2}\left(A_{2}\right)=\frac{1}{\alpha}\left(\begin{array}{ll}2 & 0 \\ 0 & 0\end{array}\right), \quad\left[m_{C}, M_{C}\right]=[0,2 / \alpha]$, $\left[m_{1}, M_{1}\right] \subset[-1.60388,4.49396]$ and $\left[m_{2}, M_{2}\right]=[0,2]$.

With respect to Remark $\square$, we obtain the following obvious corollary of Theorem $\square$.

Corollary 19 Let $\left(A_{1}, \ldots, A_{n}\right)$ be an $n$-tuple of self-adjoint operators $A_{i} \in B(H)$ with the bounds $m_{i}$ and $M_{i}, m_{i} \leq M_{i}, i=1, \ldots, n$. For some $1 \leq n_{1}<n$, we denote $m=\min \left\{m_{1}, \ldots, m_{n_{1}}\right\}$, $M=\max \left\{M_{1}, \ldots, M_{n_{1}}\right\}$. Let $\left(p_{1}, \ldots, p_{n}\right)$ be an $n$-tuple of non-negative numbers, such that $0<\sum_{i=1}^{n_{1}} p_{i}={ }_{1}<=\sum_{i=1}^{n} p_{i}$. If

$$
(m, M) \cap\left[m_{i}, M_{i}\right]=\varnothing, \quad i=n_{1}+1, \ldots, n
$$

and one of two equalities

$$
\frac{1}{1} \sum_{i=1}^{n_{1}} p_{i} A_{i}=\frac{1}{-} \sum_{i=1}^{n} p_{i} A_{i}=\frac{1}{-} \sum_{i=n_{1}+1}^{n} p_{i} A_{i}
$$

is valid, then 


$$
\frac{1}{1} \sum_{i=1}^{n_{1}} p_{i} f\left(A_{i}\right) \leq \frac{1}{-} \sum_{i=1}^{n} p_{i} f\left(A_{i}\right) \leq \frac{1}{-} \sum_{i=n_{1}+1}^{n} p_{i} f\left(A_{i}\right)
$$

holds for every continuous convex function $f: I \rightarrow \mathbb{R}$ provided that the interval $I$ contains all $m_{i}, M_{i}, i=1, \ldots, n$.

If $f: I \rightarrow \mathbb{R}$ is concave, then the reverse inequality is valid in (口)).

As a special case of Corollary $\square$ we can obtain a discrete version of Corollary $\square$ as follows.

Corollary 20 (Discrete version of Corollary $\square$ ) Let $\left(A_{1}, \ldots, A_{n}\right)$ be an $n$ - tuple of self-adjoint operators $A_{i} \in B(H)$ with the bounds $m_{i}$ and $M_{i}, m_{i} \leq M_{i}, i=1, \ldots, n$. Let $\left(\alpha_{1}, \ldots, \alpha_{n}\right)$ be an $n$ - tuple of nonnegative real numbers such that $\sum_{i=1}^{n} \alpha_{i}=1$. If

$$
\left(m_{A}, M_{A}\right) \cap\left[m_{i}, M_{i}\right]=\varnothing, \quad i=1, \ldots, n
$$

where $m_{A}$ and $M_{A}, m_{A} \leq M_{A}$, are the bounds of $A=\sum_{i=1}^{n} \alpha_{i} A_{i}$, then

$$
f\left(\sum_{i=1}^{n} \alpha_{i} A_{i}\right) \leq \sum_{i=1}^{n} \alpha_{i} f\left(A_{i}\right)
$$

holds for every continuous convex function $f: I \rightarrow \mathbb{R}$ provided that the interval $I$ contains all $m_{i}, M_{i}$.

We prove only the convex case. We define $(n+1)$-tuple of operators $\left(B_{1}, \ldots, B_{n+1}\right)$, $B_{i} \in B(H)$, by $B_{1}=A=\sum_{i=1}^{n} \alpha_{i} A_{i}$ and $B_{i}=A_{i-1}, i=2, \ldots, n+1$. Then $m_{B_{1}}=m_{A}, M_{B_{1}}=M_{A}$ are the bounds of $B_{1}$ and $m_{B_{i}}=m_{i-1}, M_{B_{i}}=M_{i-1}$ are the ones of $B_{i}, i=2, \ldots, n+1$. Also, we define $(n+1)$ - tuple of non-negative numbers $\left(p_{1}, \ldots, p_{n+1}\right)$ by $p_{1}=1$ and $p_{i}=\alpha_{i-1}, i=2, \ldots, n+1$. Then $\sum_{i=1}^{n+1} p_{i}=2$ and by using $(\square)$ we have

$$
\left(m_{B_{1}}, M_{B_{1}}\right) \cap\left[m_{B_{i}}, M_{B_{i}}\right]=\varnothing, \quad i=2, \ldots, n+1
$$

Since

$$
\sum_{i=1}^{n+1} p_{i} B_{i}=B_{1}+\sum_{i=2}^{n+1} p_{i} B_{i}=\sum_{i=1}^{n} \alpha_{i} A_{i}+\sum_{i=1}^{n} \alpha_{i} A_{i}=2 B_{1}
$$

then

$$
p_{1} B_{1}=\frac{1}{2} \sum_{i=1}^{n+1} p_{i} B_{i}=\sum_{i=2}^{n+1} p_{i} B_{i}
$$


Taking into account ( $\square$ ) and ( $\square)$, we can apply Corollary $\square$ for $n_{1}=1$ and $B_{i}, p_{i}$ as above, and we get

$$
p_{1} f\left(B_{1}\right) \leq \frac{1}{2} \sum_{i=1}^{n+1} p_{i} f\left(B_{i}\right) \leq \sum_{i=2}^{n+1} p_{i} f\left(B_{i}\right)
$$

which gives the desired inequality (־).

\section{Extension of the refined Jensen's inequality}

There is an extensive literature devoted to Jensen's inequality concerning different refinements and extensive results, see, for example [22], [23], [24], [25], [26], [27], [28], [29].

In this section we present an extension of the refined Jensen's inequality obtained in Section $\square$ and a refinement of the same inequality obtained in Section $\square$.

Theorem 21 Let $\left(A_{1}, \ldots, A_{n}\right)$ be an $n$-tuple of self-adjoint operators $A_{i} \in B(H)$ with the bounds $m_{i}$ and $M_{i}, m_{i} \leq M_{i}, i=1, \ldots, n$. Let $\left(\Phi_{1}, \ldots, \Phi_{n}\right)$ be an $n$ - tuple of positive linear mappings $\Phi_{i}: B(H) \rightarrow B(K)$, such that $\sum_{i=1}^{n_{1}} \Phi_{i}\left(1_{H}\right)=\alpha 1_{K}, \quad \sum_{i=n_{1}+1}^{n} \Phi_{i}\left(1_{H}\right)=\beta 1_{K}$, where $1 \leq n_{1}<n, \alpha, \beta>0$ and $\alpha+\beta=1$. Let $m_{L}=\min \left\{m_{1}, \ldots, m_{n_{1}}\right\}, M_{R}=\max \left\{M_{1}, \ldots, M_{n_{1}}\right\}$ and

$$
\begin{aligned}
m & =\max \left\{M_{i}: M_{i} \leq m_{L}, i \in\left\{n_{1}+1, \ldots, n\right\}\right\} \\
M & =\min \left\{m_{i}: m_{i} \geq M_{R}, i \in\left\{n_{1}+1, \ldots, n\right\}\right\}
\end{aligned}
$$

If

$$
\left(m_{L}, M_{R}\right) \cap\left[m_{i}, M_{i}\right]=\varnothing, \quad i=n_{1}+1, \ldots, n, \quad \text { and } \quad m<M
$$

and one of two equalities

$$
\frac{1}{\alpha} \sum_{i=1}^{n_{1}} \Phi_{i}\left(A_{i}\right)=\sum_{i=1}^{n} \Phi_{i}\left(A_{i}\right)=\frac{1}{\beta} \sum_{i=n_{1}+1}^{n} \Phi_{i}\left(A_{i}\right)
$$

is valid, then

$$
\begin{aligned}
\frac{1}{\alpha} \sum_{i=1}^{n_{1}} \Phi_{i}\left(f\left(A_{i}\right)\right) & \leq \frac{1}{\alpha} \sum_{i=1}^{n_{1}} \Phi_{i}\left(f\left(A_{i}\right)\right)+\beta \delta_{f} \widetilde{A} \leq \sum_{i=1}^{n} \Phi_{i}\left(f\left(A_{i}\right)\right) \\
& \leq \frac{1}{\beta} \sum_{i=n_{1}+1}^{n} \Phi_{i}\left(f\left(A_{i}\right)\right)-\alpha \delta_{f} \widetilde{A} \leq \frac{1}{\beta} \sum_{i=n_{1}+1}^{n} \Phi_{i}\left(f\left(A_{i}\right)\right)
\end{aligned}
$$


holds for every continuous convex function $f: I \rightarrow \mathbb{R}$ provided that the interval $I$ contains all $m_{i}, M_{i}, i=1, \ldots, n$, where

$$
\begin{gathered}
\delta_{f} \equiv \delta_{f}(\bar{m}, \bar{M})=f(\bar{m})+f(\bar{M})-2 f\left(\frac{\bar{m}+\bar{M}}{2}\right) \\
\left.\widetilde{A} \equiv \widetilde{A}_{A, \Phi, n_{1}, \alpha}(\bar{m}, \bar{M})=\frac{1}{2} 1_{K}-\frac{1}{\alpha(\bar{M}-\bar{m})} \sum_{i=1}^{n_{1}} \Phi_{i}|| A_{i}-\frac{\bar{m}+\bar{M}}{2} 1_{H} \mid\right)
\end{gathered}
$$

and $\bar{m} \in\left[m, m_{L}\right], \bar{M} \in\left[M_{R}, M\right], \bar{m}<\bar{M}$, are arbitrary numbers. If $f: I \rightarrow \mathbb{R}$ is concave, then the reverse inequality is valid in (口).

We prove only the convex case. Let us denote

$$
A=\frac{1}{\alpha} \sum_{i=1}^{n_{1}} \Phi_{i}\left(A_{i}\right), \quad B=\frac{1}{\beta} \sum_{i=n_{1}+1}^{n} \Phi_{i}\left(A_{i}\right), \quad C=\sum_{i=1}^{n} \Phi_{i}\left(A_{i}\right)
$$

It is easy to verify that $A=B$ or $B=C$ or $A=C$ implies $A=B=C$.

Since $f$ is convex on $[\bar{m}, \bar{M}]$ and $\left(A_{i}\right) \subseteq\left[m_{i}, M_{i}\right] \subseteq[\bar{m}, \bar{M}]$ for $i=1, \ldots, n_{1}$, it follows from Lemma $\square$ that

$$
f\left(A_{i}\right) \leq \frac{\bar{M} 1_{H}-A_{i}}{\bar{M}-\bar{m}} f(\bar{m})+\frac{A_{i}-\bar{m} 1_{H}}{\bar{M}-\bar{m}} f(\bar{M})-\delta_{f} \widetilde{A}_{i}, \quad i=1, \ldots, n_{1}
$$

holds, where $\delta_{f}=f(\bar{m})+f(\bar{M})-2 f\left(\frac{\bar{m}+\bar{M}}{2}\right)$ and $\widetilde{A}_{i}=\frac{1}{2} 1_{H}-\frac{1}{M-\bar{m}}\left|A_{i}-\frac{\bar{m}+\bar{M}}{2} 1_{H}\right|$. Applying a positive linear mapping $\Phi_{i}$ and summing, we obtain

$$
\begin{aligned}
\sum_{i=1}^{n_{1}} \Phi_{i}\left(f\left(A_{i}\right)\right) & \leq \frac{\bar{M} \alpha 1_{K}-\sum_{i=1}^{n_{1}} \Phi_{i}\left(A_{i}\right)}{\bar{M}-\bar{m}} f(\bar{m})+\frac{\sum_{i=1}^{n_{1}} \Phi_{i}\left(A_{i}\right)-\bar{m} \alpha 1_{K}}{\bar{M}-\bar{m}} f(\bar{M}) \\
& -\delta_{f}\left(\frac{\alpha}{2} 1_{K}-\bar{M}-\bar{m} \sum_{i=1}^{n_{1}} \Phi_{i}\left(\left|A_{i}-\frac{\bar{m}+\bar{M}}{2} 1_{H}\right|\right)\right)
\end{aligned}
$$

since $\sum_{i=1}^{n_{1}} \Phi_{i}\left(1_{H}\right)=\alpha 1_{K}$. It follows that

$$
\frac{1}{\alpha} \sum_{i=1}^{n_{1}} \Phi_{i}\left(f\left(A_{i}\right)\right) \leq \frac{\bar{M} 1_{K}-A}{\bar{M}-\bar{m}} f(\bar{m})+\frac{A-\bar{m} 1_{K}}{\bar{M}-\bar{m}} f(\bar{M})-\delta_{f} \widetilde{A}
$$

where $\widetilde{A}=\frac{1}{2} 1_{K}-\frac{1}{\alpha(M-\bar{m})} \sum_{i=1}^{n_{1}} \Phi_{i}\left(\left|A_{i}-\frac{\bar{m}+\bar{M}}{2} 1_{H}\right|\right)$. 
Additionally, since $f$ is convex on all $\left[m_{i}, M_{i}\right]$ and $(\bar{m}, \bar{M}) \cap\left[m_{i}, M_{i}\right]=\varnothing, i=n_{1}+1, \ldots, n$, then

$$
f\left(A_{i}\right) \geq \frac{\bar{M} 1_{H}-A_{i}}{\bar{M}-\bar{m}} f(\bar{m})+\frac{A_{i}-\bar{m} 1_{H}}{\bar{M}-\bar{m}} f(\bar{M}), \quad i=n_{1}+1, \ldots, n
$$

It follows

$$
\frac{1}{\beta} \sum_{i=n_{1}+1}^{n} \Phi_{i}\left(f\left(A_{i}\right)\right)-\delta_{f} \widetilde{A} \geq \frac{\bar{M} 1_{K}-B}{\bar{M}-\bar{m}} f(\bar{m})+\frac{B-\bar{m} 1_{K}}{\bar{M}-\bar{m}} f(\bar{M})-\delta_{f} \widetilde{A}
$$

Combining (ㅁ) and (ㅁ) and taking into account that $A=B$, we obtain

$$
\frac{1}{\alpha} \sum_{i=1}^{n_{1}} \Phi_{i}\left(f\left(A_{i}\right)\right) \leq \frac{1}{\beta} \sum_{i=n_{1}+1}^{n} \Phi_{i}\left(f\left(A_{i}\right)\right)-\delta_{f} \widetilde{A}
$$

Next, we obtain

$$
\begin{aligned}
& \frac{1}{\alpha} \sum_{i=1}^{n_{1}} \Phi_{i}\left(f\left(A_{i}\right)\right) \\
= & \sum_{i=1}^{n_{1}} \Phi_{i}\left(f\left(A_{i}\right)\right)+\frac{\beta}{\alpha} \sum_{i=1}^{n_{1}} \Phi_{i}\left(f\left(A_{i}\right)\right) \quad(\text { by } \alpha+\beta=1) \\
\leq & \sum_{i=1}^{n_{1}} \Phi_{i}\left(f\left(A_{i}\right)\right)+\sum_{i=n_{1}+1}^{n} \Phi_{i}\left(f\left(A_{i}\right)\right)-\beta \delta_{f} \widetilde{A} \quad(\text { by }()) \\
\leq & \left.\frac{\alpha}{\beta} \sum_{i=n_{1}+1}^{n} \Phi_{i}\left(f\left(A_{i}\right)\right)-\alpha \delta_{f} \widetilde{A}+\sum_{i=n_{1}+1}^{n} \Phi_{i}\left(f\left(A_{i}\right)\right)-\beta \delta_{f} \widetilde{A} \quad \text { (by }()\right) \\
= & \frac{1}{\beta} \sum_{i=n_{1}+1}^{n} \Phi_{i}\left(f\left(A_{i}\right)\right)-\delta_{f} \widetilde{A} \quad(\text { by } \alpha+\beta=1)
\end{aligned}
$$

which gives the following double inequality

$$
\frac{1}{\alpha} \sum_{i=1}^{n_{1}} \Phi_{i}\left(f\left(A_{i}\right)\right) \leq \sum_{i=1}^{n} \Phi_{i}\left(f\left(A_{i}\right)\right)-\beta \delta_{f} \widetilde{A} \leq \frac{1}{\beta} \sum_{i=n_{1}+1}^{n} \Phi_{i}\left(f\left(A_{i}\right)\right)-\delta_{f} \widetilde{A}
$$

Adding $\beta \delta_{f} \widetilde{A}$ in the above inequalities, we get

$$
\frac{1}{\alpha} \sum_{i=1}^{n_{1}} \Phi_{i}\left(f\left(A_{i}\right)\right)+\beta \delta_{f} \widetilde{A} \leq \sum_{i=1}^{n} \Phi_{i}\left(f\left(A_{i}\right)\right) \leq \frac{1}{\beta} \sum_{i=n_{1}+1}^{n} \Phi_{i}\left(f\left(A_{i}\right)\right)-\alpha \delta_{f} \widetilde{A}
$$




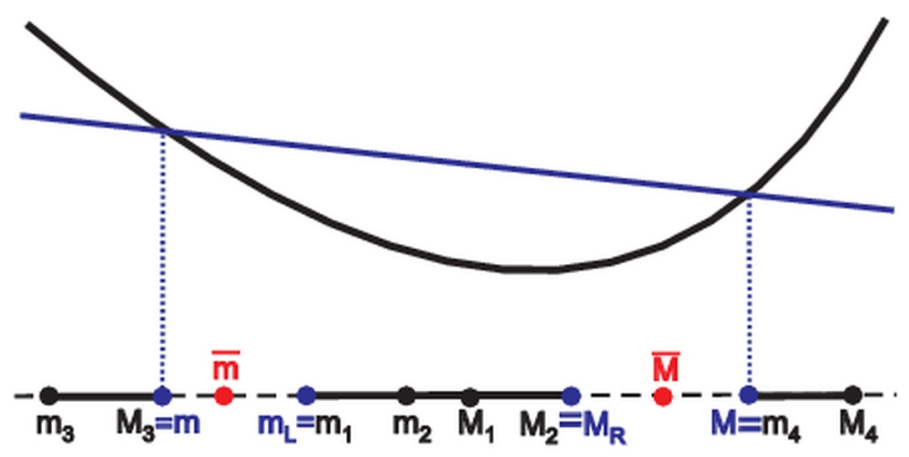

Figure 3. An example a convex function and the bounds of four operators

Now, we remark that $\delta_{f} \geq 0$ and $\widetilde{A} \geq 0$. (Indeed, since $f$ is convex, then $f((\bar{m}+\bar{M}) / 2) \leq(f(\bar{m})+f(\bar{M})) / 2$, which implies that $\delta_{f} \geq 0$. Also, since

$$
\left(A_{i}\right) \subseteq[\bar{m}, \bar{M}] \Rightarrow\left|A_{i}-\frac{\bar{M}+\bar{m}}{2} 1_{H}\right| \leq \frac{\bar{M}-\bar{m}}{2} 1_{H}, \quad i=1, \ldots, n_{1}
$$

then

$$
\sum_{i=1}^{n_{1}} \Phi_{i}\left(\left|A_{i}-\frac{\bar{M}+\bar{m}}{2} 1_{H}\right|\right) \leq \frac{\bar{M}-\bar{m}}{2} \alpha 1_{K}
$$

which gives

$$
\left.0 \leq \frac{1}{2} 1_{K}-\frac{1}{\alpha(\bar{M}-\bar{m})} \sum_{i=1}^{n_{1}} \Phi_{i}\left(\left|A_{i}-\frac{\bar{M}+\bar{m}}{2} 1_{H}\right|\right)=\widetilde{A}\right)
$$

Consequently, the following inequalities

$$
\begin{aligned}
& \frac{1}{\alpha} \sum_{i=1}^{n_{1}} \Phi_{i}\left(f\left(A_{i}\right)\right) \leq \frac{1}{\alpha} \sum_{i=1}^{n_{1}} \Phi_{i}\left(f\left(A_{i}\right)\right)+\beta \delta_{f} \widetilde{A} \\
& \frac{1}{\beta} \sum_{i=n_{1}+1}^{n} \Phi_{i}\left(f\left(A_{i}\right)\right)-\alpha \delta_{f} \widetilde{A} \leq \frac{1}{\beta} \sum_{i=n_{1}+1}^{n} \Phi_{i}\left(f\left(A_{i}\right)\right)
\end{aligned}
$$

hold, which with (־) proves the desired series inequalities (ㅁ). 1.05

Example 22 We observe the matrix case of Theorem $\square$ for $f(t)=t^{4}$, which is the convex function but not operator convex, $n=4, n_{1}=2$ and the bounds of matrices as in Fig. 3 .

We show an example such that 


$$
\begin{gathered}
\frac{1}{\alpha}\left(\Phi_{1}\left(A_{1}^{4}\right)+\Phi_{2}\left(A_{2}^{4}\right)\right)<\frac{1}{\alpha}\left(\Phi_{1}\left(A_{1}^{4}\right)+\Phi_{2}\left(A_{2}^{4}\right)\right)+\beta \delta_{f} \widetilde{A} \\
<\Phi_{1}\left(A_{1}^{4}\right)+\Phi_{2}\left(A_{2}^{4}\right)+\Phi_{3}\left(A_{3}^{4}\right)+\Phi_{4}\left(A_{4}^{4}\right) \\
<\frac{1}{\beta}\left(\Phi_{3}\left(A_{3}^{4}\right)+\Phi_{4}\left(A_{4}^{4}\right)\right)-\alpha \delta_{f} \widetilde{A}<\frac{1}{\beta}\left(\Phi_{3}\left(A_{3}^{4}\right)+\Phi_{4}\left(A_{4}^{4}\right)\right)
\end{gathered}
$$

holds, where $\delta_{f}=\bar{M}^{4}+\bar{m}^{4}-(\bar{M}+\bar{m})^{4} 8$ and

$$
\widetilde{A}=\frac{1}{2} I_{2}-\frac{1}{\alpha(\bar{M}-\bar{m})}\left(\Phi_{1}\left(\left|A_{1}-\frac{\bar{M}+\bar{m}}{2} I_{h}\right|\right)+\Phi_{2}\left(\left|A_{2}-\frac{\bar{M}+\bar{m}}{2} I_{3}\right|\right)\right)
$$

We define mappings $\Phi_{i}: M_{3}(\mathbb{C}) \rightarrow M_{2}(\mathbb{C})$ as follows: $\Phi_{i}\left(\left(a_{j k}\right)_{1 \leq j, k \leq 3}\right)=\frac{1}{4}\left(a_{j k}\right)_{1 \leq j, k \leq 2^{\prime}}$ $i=1, \ldots, 4$. Then $\sum_{i=1}^{4} \Phi_{i}\left(I_{3}\right)=I_{2}$ and $\alpha=\beta=\frac{1}{2}$.

Let

$$
A_{1}=2\left(\begin{array}{ccc}
2 & 9 / 8 & 1 \\
9 / 8 & 2 & 0 \\
1 & 0 & 3
\end{array}\right), A_{2}=3\left(\begin{array}{ccc}
2 & 9 / 8 & 0 \\
9 / 8 & 1 & 0 \\
0 & 0 & 2
\end{array}\right), A_{3}=-3\left(\begin{array}{ccc}
4 & 1 / 2 & 1 \\
1 / 2 & 4 & 0 \\
1 & 0 & 2
\end{array}\right), A_{4}=12\left(\begin{array}{ccc}
5 / 3 & 1 / 2 & 0 \\
1 / 2 & 3 / 2 & 0 \\
0 & 0 & 3
\end{array}\right)
$$

Then $m_{1}=1.28607, M_{1}=7.70771, m_{2}=0.53777, M_{2}=5.46221, m_{3}=-14.15050, M_{3}=-4.71071$, $m_{4}=12.91724, M_{4}=36$., so $m_{L}=m_{2}, M_{R}=M_{1}, m=M_{3}$ and $M=m_{4}$ (rounded to five decimal places). Also,

$$
\frac{1}{\alpha}\left(\Phi_{1}\left(A_{1}\right)+\Phi_{2}\left(A_{2}\right)\right)=\frac{1}{\beta}\left(\Phi_{3}\left(A_{3}\right)+\Phi_{4}\left(A_{4}\right)\right)=\left(\begin{array}{cc}
4 & 9 / 4 \\
9 / 4 & 3
\end{array}\right)
$$

and

$$
\begin{aligned}
A_{f} \equiv \frac{1}{\alpha}\left(\Phi_{1}\left(A_{1}^{4}\right)+\Phi_{2}\left(A_{2}^{4}\right)\right) & =\left(\begin{array}{cc}
989.00391 & 663.46875 \\
663.46875 & 526.12891
\end{array}\right) \\
C_{f} \equiv \Phi_{1}\left(A_{1}^{4}\right)+\Phi_{2}\left(A_{2}^{4}\right)+\Phi_{3}\left(A_{3}^{4}\right)+\Phi_{4}\left(A_{4}^{4}\right) & =\left(\begin{array}{cc}
68093.14258 & 48477.98437 \\
48477.98437 & 51335.39258
\end{array}\right) \\
B_{f} \equiv \frac{1}{\beta}\left(\Phi_{3}\left(A_{3}^{4}\right)+\Phi_{4}\left(A_{4}^{4}\right)\right) & =\left(\begin{array}{cc}
135197.28125 & 96292.5 \\
96292.5 & 102144.65625
\end{array}\right)
\end{aligned}
$$

Then

$$
A_{f}<C_{f}<B_{f}
$$


holds (which is consistent with (ㅁ)).

We will choose three pairs of numbers $(\bar{m}, \bar{M}), \quad \bar{m} \in[-4.71071,0.53777]$, $\bar{M} \in[7.70771,12.91724]$ as follows

i) $\bar{m}=m_{L}=0.53777, \bar{M}=M_{R}=7.70771$, then

$\widetilde{\Delta}_{1}=\beta \delta_{f} \widetilde{A}=0.5 \cdot 2951.69249 \cdot\left(\begin{array}{ll}0.15678 & 0.09030 \\ 0.09030 & 0.15943\end{array}\right)=\left(\begin{array}{ll}231.38908 & 133.26139 \\ 133.26139 & 235.29515\end{array}\right)$

ii) $\bar{m}=m=-4.71071, \bar{M}=M=12.91724$, then

$\widetilde{\Delta}_{2}=\beta \delta_{f} \widetilde{A}=0.5 \cdot 27766.07963 \cdot\left(\begin{array}{ll}0.36022 & 0.03573 \\ 0.03573 & 0.36155\end{array}\right)=\left(\begin{array}{cc}5000.89860 & 496.04498 \\ 496.04498 & 5019.50711\end{array}\right)$

iii) $\bar{m}=-1, \bar{M}=10$, then

$\widetilde{\Delta}_{3}=\beta \delta_{f} \widetilde{A}=0.5 \cdot 9180.875 \cdot\left(\begin{array}{cc}0.28203 & 0.08975 \\ 0.08975 & 0.27557\end{array}\right)=\left(\begin{array}{cc}1294.66 & 411.999 \\ 411.999 & 1265 .\end{array}\right)$

New, we obtain the following improvement of $(\square)$ (see (口))

\section{Table 1.}

Using Theorem $\square$ we get the following result.

Corollary 23 Let the assumptions of Theorem $\square$ hold. Then

$$
\frac{1}{\alpha} \sum_{i=1}^{n_{1}} \Phi_{i}\left(f\left(A_{i}\right)\right) \leq \frac{1}{\alpha} \sum_{i=1}^{n_{1}} \Phi_{i}\left(f\left(A_{i}\right)\right)+\gamma_{1} \delta_{f} \widetilde{A} \leq \frac{1}{\beta} \sum_{i=n_{1}+1}^{n} \Phi_{i}\left(f\left(A_{i}\right)\right)
$$

and

$$
\frac{1}{\alpha} \sum_{i=1}^{n_{1}} \Phi_{i}\left(f\left(A_{i}\right)\right) \leq \frac{1}{\beta} \sum_{i=n_{1}+1}^{n} \Phi_{i}\left(f\left(A_{i}\right)\right)-\gamma_{2} \delta_{f} \widetilde{A} \leq \frac{1}{\beta} \sum_{i=n_{1}+1}^{n} \Phi_{i}\left(f\left(A_{i}\right)\right)
$$

holds for every $\gamma_{1}, \gamma_{2}$ in the close interval joining $\alpha$ and $\beta$, where $\delta_{f}$ and $\widetilde{A}$ are defined by (ㅁ).

Adding $\alpha \delta_{f} \widetilde{A}$ in (口) and noticing $\delta_{f} \widetilde{A} \geq 0$, we obtain

$$
\frac{1}{\alpha} \sum_{i=1}^{n_{1}} \Phi_{i}\left(f\left(A_{i}\right)\right) \leq \frac{1}{\alpha} \sum_{i=1}^{n_{1}} \Phi_{i}\left(f\left(A_{i}\right)\right)+\alpha \delta_{f} \widetilde{A} \leq \frac{1}{\beta} \sum_{i=n_{1}+1}^{n} \Phi_{i}\left(f\left(A_{i}\right)\right)
$$

Taking into account the above inequality and the left hand side of ( $\square)$ we obtain ( $\square$ ). 
Similarly, subtracting $\beta \delta_{f} \widetilde{A}$ in (口) we obtain (口).

Remark 24 We can obtain extensions of inequalities which are given in Remark $\square$ and $\square$. Also, we can obtain a special case of Theorem $\square$ with the convex combination of operators $A_{i}$ putting $\Phi_{i}(B)=\alpha_{i} B$, for $i=1, \ldots, n$, similarly as in Corollary $\square$. Finally, applying this result, we can give another proof of Corollary $\square$. The interested reader can see the details in [30].

\section{Author details}

Jadranka Mićić ${ }^{1}$ and Josip Pečarić ${ }^{2}$

Faculty of Mechanical Engineering and Naval Architecture, University of Zagreb, Ivana Lučića 5, 10000 Zagreb,, Croatia

Faculty of Textile Technology, University of Zagreb, Prilaz baruna Filipovića 30, 10000 Zagreb, Croatia

\section{References}

[1] Davis C (1957) A Schwarz inequality for convex operator functions. Proc. Amer. Math. Soc. 8: 42-44.

[2] Choi M.D (1974) A Schwarz inequality for positive linear maps on C *algebras. Illinois J. Math. 18: 565-574.

[3] Hansen F, Pedersen G.K (1982) Jensen's inequality for operators and Löwner's theorem. Math. Ann. 258: 229-241.

[4] Mond B, Pečarić J (1995) On Jensen's inequality for operator convex functions. Houston J. Math. 21: 739-754.

[5] Hansen F, Pedersen G.K (2003) Jensen's operator inequality. Bull. London Math. Soc. 35: 553-564.

[6] Mond B, Pečarić J (1994) Converses of Jensen's inequality for several operators, Rev. Anal. Numér. Théor. Approx. 23: 179-183.

[7] Mond B, Pečarić J.E (1993) Converses of Jensen's inequality for linear maps of operators, An. Univ. Vest Timiş. Ser. Mat.-Inform. XXXI 2: 223-228.

[8] Furuta T (1998) Operator inequalities associated with Hölder-McCarthy and Kantorovich inequalities. J. Inequal. Appl. 2: 137-148.

[9] Mićić J, Seo Y, Takahasi S.E, Tominaga M (1999) Inequalities of Furuta and MondPečarić. Math. Inequal. Appl. 2: 83-111. 
[10] Mićić J, Pečarić J, Seo Y, Tominaga M (2000) Inequalities of positive linear maps on Hermitian matrices. Math. Inequal. Appl. 3: 559-591.

[11] Fujii M, Mićić Hot J, Pečarić J, Seo Y (2012) Recent Developments of Mond-Pečarić method in Operator Inequalities. Zagreb: Element. 320 p. In print.

[12] Fujii J.I (2011) An external version of the Jensen operator inequality. Sci. Math. Japon. Online e-2011: 59-62.

[13] Fujii J.I, Pečarić J, Seo Y (2012) The Jensen inequality in an external formula. J. Math. Inequal. Accepted for publication.

[14] Hansen F, Pečarić J, Perić I (2007) Jensen's operator inequality and it's converses. Math. Scand. 100: 61-73.

[15] Furuta T, Mićić Hot J, Pečarić J, Seo Y (2005) Mond-Pečarić Method in Operator Inequalities. Zagreb: Element. 262 p.

[16] Mićić J, Pečarić J, Seo Y (2010) Converses of Jensen's operator inequality. Oper. Matrices 4: 385-403.

[17] Mićić J, Pavić Z, Pečarić J (2011) Some better bounds in converses of the Jensen operator inequality. Oper. Matrices. In print.

[18] Mićić J, Pavić Z, Pečarić J (2011) Jensen's inequality for operators without operator convexity. Linear Algebra Appl. 434: 1228-1237.

[19] Mićić J, Pečarić J, Perić J (2012) Refined Jensen's operator inequality with condition on spectra. Oper. Matrices. Accepted for publication.

[20] Mitrinović D.S, Pečarić J.E, Fink A.M (1993) Classical and New Inequalities in Analysis. Dordrecht-Boston-London: Kluwer Acad. Publ. 740 p.

[21] Mićić J, Pavić Z, Pečarić J (2011) Extension of Jensen's operator inequality for operators without operator convexity. Abstr. Appl. Anal. 2011: 1-14.

[22] Abramovich S, Jameson G, Sinnamon G (2004) Refining Jensen's inequality, Bull. Math. Soc. Sci. Math. Roumanie (N.S.) 47: 3-14.

[23] Dragomir S.S (2010) A new refinement of Jensen's inequality in linear spaces with applications. Math. Comput. Modelling 52: 1497-1505.

[24] Khosravi M, Aujla J.S, Dragomir S.S, Moslehian M.S (2011) Refinements of Choi-Davis-Jensen's inequality. Bull. Math. Anal. Appl. 3: 127-133.

[25] Moslehian M.S (2009) Operator extensions of Hua's inequality. Linear Algebra Appl. 430: 1131-1139.

[26] Rooin J (2005) A refinement of Jensen's inequality, J. Ineq. Pure and Appl. Math. 6. 2. Art. 38: 4 p. 
[27] Srivastava H.M, Xia Z.G, Zhang Z.H (2011) Some further refinements and extensions of the Hermite-Hadamard and Jensen inequalities in several variables. Math. Comput. Modelling 54: 2709-2717.

[28] Xiao Z.G, Srivastava H.M, Zhang Z.H (2010) Further refinements of the Jensen inequalities based upon samples with repetitions. Math. Comput. Modelling 51: 592-600.

[29] Wang L.C, Ma X.F, Liu L.H (2009) A note on some new refinements of Jensen's inequality for convex functions. J. Inequal. Pure Appl. Math. 10. 2. Art. $48: 6$ p.

[30] Mićić J, Pečarić J, Perić J (2012) Extension of the refined Jensen's operator inequality with condition on spectra. Ann. Funct. Anal. 3: 67-85. 